\title{
TU/e EmonOWEN

\section{Analysis of influences of pressure anisotropies on the 3D MHD equilibrium in LHD}

Citation for published version (APA):

Romba, T., Suzuki, Y., \& Proll, J. H. E. (2021). Analysis of influences of pressure anisotropies on the 3D MHD equilibrium in LHD. Physics of Plasmas, 28(4), [042504]. https://doi.org/10.1063/5.0033807

DOI:

$10.1063 / 5.0033807$

Document status and date:

Published: 01/04/2021

\section{Document Version:}

Accepted manuscript including changes made at the peer-review stage

\section{Please check the document version of this publication:}

- A submitted manuscript is the version of the article upon submission and before peer-review. There can be important differences between the submitted version and the official published version of record. People interested in the research are advised to contact the author for the final version of the publication, or visit the $\mathrm{DOI}$ to the publisher's website.

- The final author version and the galley proof are versions of the publication after peer review.

- The final published version features the final layout of the paper including the volume, issue and page numbers.

Link to publication

\section{General rights}

Copyright and moral rights for the publications made accessible in the public portal are retained by the authors and/or other copyright owners and it is a condition of accessing publications that users recognise and abide by the legal requirements associated with these rights.

- Users may download and print one copy of any publication from the public portal for the purpose of private study or research.

- You may not further distribute the material or use it for any profit-making activity or commercial gain

- You may freely distribute the URL identifying the publication in the public portal.

If the publication is distributed under the terms of Article 25fa of the Dutch Copyright Act, indicated by the "Taverne" license above, please follow below link for the End User Agreement:

www.tue.nl/taverne

Take down policy

If you believe that this document breaches copyright please contact us at:

openaccess@tue.nl

providing details and we will investigate your claim. 
Analysis of influences of pressure anisotropies on the 3D MHD equilibrium in LHD

Analysis of influences of pressure anisotropies on the 3D MHD equilibrium in LHD

T. Romba, ${ }^{1, \text { a) }}$ Y. Suzuki, ${ }^{2,3}$ and J.H.E. Proll ${ }^{1}$

${ }^{1)}$ Eindhoven University of Technology, PO Box 513, 5600 MB Eindhoven, The Netherlands

${ }^{2)}$ National Institute for Fusion Science, National Institutes of Natural Sciences, Toki 509-5292, Japan

${ }^{3)}$ The Graduate University for Advanced Studies, SOKENDAI, Toki 509-5292, Japan

(Dated: 18 March 2021)

3D equilibria with an anisotropic pressure component in the Large Helical Device are analysed with respect to their magnetic axis locations. The anisotropic extension of the 3D equilibrium solver VMEC, ANIMEC, is used to compute fixed-boundary plasma equilibria based on a Bi-Maxwellian distribution function describing the anisotropic particles. Different heating scenarios are assessed by means of parallel and perpendicular pressure anisotropies with different radial anisotropic pressure profiles imposed. A theoretical predicted scaling of the magnetic axis location with the auxiliary parameter $\beta_{e q}$ as predicted for classical stellarators and heliotrons by Hitchon [Nuclear Fusion 23, 383(1983)] is found to be applicable to the Large Helical Device in case of a flat hot-particle profile for parallel or weak perpendicular dominated anisotropies with $\beta_{\perp} / \beta_{\|} \leq 2$. For strong perpendicular or non-flat hot-particle profiles, a deviation from the predicted scaling of the magnetic axis location is found. While center peaked profiles show a stronger shift of the magnetic axis, edge peaked profiles show no significant change of its radial location. High critical magnetic fields are identified as a necessary condition for strong perpendicular anisotropies. The observed deviations are ascribed to the magnetic field structure and negative pressure gradients. The invalidity of the theoretical predictions in case of certain configurations is found to be caused by higher order terms in the pressure components which are not accounted for by the ordering on which the theory is based.

a)Electronic mail: t.romba@student.tue.nl 
Analysis of influences of pressure anisotropies on the 3D MHD equilibrium in LHD

\section{INTRODUCTION}

Investigations of plasmas of high ratios of plasma pressure to magnetic pressure, $\beta$, are a crucial step on the path to commercially viable nuclear fusion reactors. In the Large Helical Device (LHD), high $\beta$ operation with a spatial averaged $\beta$ up to $4 \%^{1}$ is achieved by operation of low density plasmas at low magnetic fields ${ }^{2}$. Such plasmas are sustained by tangential neutral beam injection and have a low collisionality and, subsequently, a long slowing down time of the hot injected particles. With the thermal plasma pressure being low due to the low density, fast particles have a significant contribution to the total plasma pressure in those high $\beta$ scenarios. Monte-Carlo simulations estimated that up to $30 \%$ of the total volume-averaged beta can be ascribed to an anisotropic pressure component in those scenarios ${ }^{3}$.

To reach thermonuclear conditions, auxiliary heating systems are necessary. As these heat the plasma particles non-uniformly but with certain preferential directions, an anisotropic population with an associated pressure component is created within the plasma. The anisotropic character of this population causes it to have different influences onto the plasma equilibrium than the isotropic bulk plasma has. These influences may change the magnetic axis location, the central value of the rotational transform as well as the local magnetic field strength.

The 3D equilibrium solver $\mathrm{VMEC}^{4}$ is a renowned code to determine plasma equilibria in stellarator geometry and was expanded by Cooper et al. to treat anisotropic pressure components in its extension ANIMEC ${ }^{5}$. Pressure anisotropies are either described by a Bi-Maxwellian distribution function $^{6}$ or a modified slowing down distribution ${ }^{7,8}$. While the Bi-Maxwellian distribution was shown to be applicable for parallel plasma anisotropies caused by tangential NBI in LHD 9 and perpendicular pressure anisotropies caused by ICRH in $\mathrm{JET}^{10}$, the modified slowing down distribution function, expected to describe NBI heated plasmas for any injection angle ${ }^{5}$, has been tested ${ }^{11}$ but was not used for systematic studies of pressure anisotropies yet. In 9, the Bi-Maxwellian distribution function implemented in ANIMEC was used to investigate plasmas sustained by tangential NBI with fast particles being deposited over the whole plasma region. This study found a match of simulated and theoretically predicted magnetic axis locations for parallel pressure anisotropies in case of a flat hot-particle profile. An extension of these investigations by analysing perpendicular dominated anisotropic plasmas as well as non-flat hot-particle profiles has not been performed yet.

Using fixed-boundary equilibrium calculations and the Bi-Maxwellian distribution function implemented in ANIMEC, the influences of parallel and perpendicular pressure anisotropies onto 
Analysis of influences of pressure anisotropies on the 3D MHD equilibrium in LHD

the plasma equilibrium configuration in LHD are investigated. Heating scenarios giving rise to fast particles at different radial locations are assessed by different hot-particle pressure profiles.

Section II covers the theoretical background of 3D equilibria with an emphasis on theoretical predictions of the radial magnetic axis position changed by the Shafranov-shift. Section III covers the theory behind the equilibrium solver VMEC and its anisotropic extension ANIMEC. Section IV covers the results obtained for flat, edge-peaked, and center-peaked hot-particle pressure profiles as well as a reassessment of the theoretical framework introduced in section II. Section V summarizes the results and points out arising questions for future research.

\section{THEORETICAL TREATMENT OF ANISOTROPIC PLASMAS}

The theoretical quantification of influences of anisotropic pressure components onto a plasma equilibrium is done by means of assigning a spatially averaged $\beta$ and comparing central plasma parameters, such as the magnetic axis location, to results obtained in isotropic cases of comparable $\beta$. In context of pressure anisotropies, two different $\beta$ definitions may be of use. Based on the plasma energy, using the principle of equipartition, one can assign a total beta value, $\beta_{t o t}$, to a plasma

$$
\beta_{t o t}=\frac{\frac{1}{3} \int\left(p_{\|}+2 p_{\perp}\right) \mathrm{dV}}{\int \frac{B^{2}}{2 \mu_{0}} \mathrm{dV}},
$$

where $p_{\|}$and $p_{\perp}$ are the parallel and perpendicular pressures, respectively, $B$ is the magnetic field strength, and $\mu_{0}$ is the vacuum permeability. The integration is carried out over the whole plasma volume to obtain a spatially averaged quantity.

Alternatively, $\beta$ can be based on an analytical treatment of anisotropic plasmas which aims to predict a certain parameter scaling as done by Hitchon et al. ${ }^{12,13}$. This framework uses a low beta ordering $\beta \sim O\left(\varepsilon^{2}\right)^{14}$ and the pressure tensor from the hydromagnetic equations $\overleftrightarrow{p}=p_{\perp} I+\left(p_{\|}-\right.$ $\left.p_{\perp}\right) \boldsymbol{b} \boldsymbol{b}^{15}$ where $\varepsilon$ is the inverse aspect ratio, $I$ the identity matrix, and $\boldsymbol{b}$ is the unit vector along the magnetic field lines. In this ordering, which was initially proposed for classical stellarators and heliotrons, quantities are reduced to their flux-surface averages and an equivalent beta, $\beta_{\text {eq }}$, is derived

$$
\beta_{e q}=\frac{\frac{1}{2} \int\left(p_{\|}+p_{\perp}\right) \mathrm{dV}}{\int \frac{B^{2}}{2 \mu_{0}} \mathrm{dV}} .
$$

This parameter is defined such that the same radial shift of the magnetic axis is present for anisotropic plasmas of $\beta_{e q}$ as it is for isotropic plasma of same $\beta$. Note that the two $\beta$ defini- 
Analysis of influences of pressure anisotropies on the 3D MHD equilibrium in LHD

tions are equal in the isotropic case and that they relate like

$$
\beta_{e q}=\frac{3}{2} \beta_{t o t}-\frac{1}{2} \beta_{\perp}=\frac{3}{2} \beta_{t o t} \frac{1+\beta_{\perp} / \beta_{\|}}{1+2 \beta_{\perp} / \beta_{\|}},
$$

where

$$
\begin{aligned}
& \beta_{\perp}=\frac{\int p_{\perp} \mathrm{dV}}{\int \frac{B^{2}}{2 \mu_{0}} \mathrm{dV}} \\
& \beta_{\|}=\frac{\int p_{\|} \mathrm{dV}}{\int \frac{B^{2}}{2 \mu_{0}} \mathrm{dV}} .
\end{aligned}
$$

In the flux-surface-averaged treatment, the flux-surface averages of the parallel and perpendicular pressure, $P_{\|}$and $P_{\perp}$, are defined. The Pfirsch-Schlüter current then follows to ${ }^{12,16}$

$$
j_{p s}=\frac{\rho}{b^{*}}\left(P_{\|}^{\prime}+P_{\perp}^{\prime}\right) \cos \theta
$$

where $\rho=r / a$ is the normalized radial coordinate, ' is the radial derivative, $\theta$ is the poloidal angle, and $b^{*}$ is the effective mean poloidal field as defined in Eq. (17) in 17.

Any such current running in the plasma gives rise to a magnetic field however. With such a plasma based magnetic field component, $\boldsymbol{B}_{p l}$, the total magnetic field, $\boldsymbol{B}_{t o t}$, can be expressed as a linear combination of vacuum and plasma related field strength

$$
\boldsymbol{B}_{t o t}=\boldsymbol{B}_{v a c}+\boldsymbol{B}_{p l}
$$

where $\boldsymbol{B}_{v a c}$ is the vacuum magnetic field caused by the currents through the external field coils. Using this relation, the knowledge of the field strengths in a given equilibrium, as well as the vacuum field strength, one can determine the magnetic field component which arises due to the plasma current. In the following, the vertical magnetic field component which can be assigned to the plasma current is denoted with $B_{z, p l}$.

To monitor the stability of a plasma configuration, the fire hose stability criterion ${ }^{18}$

$$
\sigma=\frac{1}{\mu_{0}}-\left.\frac{1}{B} \frac{\partial p_{\|}}{\partial B}\right|_{s}=\frac{1}{\mu_{0}}-\frac{p_{\|}-p_{\perp}}{B^{2}}>0,
$$

as well as the mirror stability criterion ${ }^{18}$

$$
\tau=\left.\frac{\partial(\sigma B)}{\partial B}\right|_{s}=\frac{1}{\mu_{0}}+\left.\frac{1}{B} \frac{\partial p_{\perp}}{\partial B}\right|_{s}>0,
$$


Analysis of influences of pressure anisotropies on the 3D MHD equilibrium in LHD

need to be fulfilled. In both expressions, $s$ is a radial index proportional to the enclosed toroidal flux $2 \pi \Phi$ where $\Phi$ is the toroidal magnetic flux function.

In the framework of the stability criteria in Eqs. (7) and (8) and using the parameters defined beforehand, a numerical framework for a 3D equilibrium solver with an anisotropic particle distribution is introduced next.

\section{NUMERICAL MODEL}

The MHD equilibrium code $\mathrm{VMEC}^{4}$ numerically computes a 3D equilibrium in a set isotropic plasma configuration by means of a variational principle which minimizes the plasma energy $W^{19}$

$$
W=\int\left(\frac{B^{2}}{2 \mu_{0}}+\frac{p}{\Gamma-1}\right) \mathrm{dV}
$$

where $p$ is the isotropic plasma pressure, and $\Gamma$ is the ratio of the specific heats. In case of an anisotropic pressure component, the definition of the plasma energy in the variational principle is ${ }^{7}$

$$
W=\int\left(\frac{B^{2}}{2 \mu_{0}}+\frac{p_{\|}(s, B)}{\Gamma-1}\right) \mathrm{dV},
$$

where $p_{\|}(s, B)$ denotes the total parallel pressure at a flux-surface $s$, dependent on the local magnetic field strength $B$. Note that by introducing a $B$-dependency to $p_{\|}$, the pressure is no longer a flux-surface quantity. In plasmas with anisotropic populations, the total pressure is given by the sum of the thermal bulk pressure and the pressure imposed by the anisotropic species. The profile of the latter is specified by an amplitude factor $p_{h}(s)$, giving rise to the total parallel pressure being of the form

$$
p_{\|}(s, B)=m(s)\left[1+p_{h}(s) H(s, B)\right],
$$

where $m(s)$ denotes the plasma mass and $H(s, B)$ is a factor describing local pressure variations around a flux-surface due to high energetic particles. In the isotropic limiting case, Eq. (11) reduces to its isotropic component only, making $p_{\|}$a flux-surface constant again. Note that an imposed hot-particle profile in Eq. (11) does not influence the total parallel pressure directly but only via the product $m(s) p_{h}(s)$, therefore, the anisotropic part only enters in form of a multiple of the thermal pressure profile. This allows the anisotropic profile to deviate from the imposed radial profile.

Instead of implementing a similar expression for the total pressure perpendicular to the magnetic field lines, $p_{\perp}(s, B)$, ANIMEC uses the force balance parallel to $\mathbf{B}$ to determine the second 
Analysis of influences of pressure anisotropies on the 3D MHD equilibrium in LHD

pressure component ${ }^{7}$

$$
p_{\perp}(s, B)=p_{\|}(s, B)-\left.B \frac{\partial p_{\|}}{\partial B}\right|_{s} .
$$

In analogy to the Maxwell distribution used to describe isotropic particles, anisotropic populations are described by solutions of appropriate differential equations. As the lowest order solution of the Fokker-Planck equation $\mathbf{B} \cdot \nabla \mathscr{F}_{h}=0$, a Bi-Maxwellian distribution $\mathscr{F}_{h}$ was found to yield appropriate results for plasmas with parallel anisotropies sustained by tangential $\mathrm{NBI}^{9}$ as well as plasmas with perpendicular anisotropies heated by $\mathrm{ICRH}^{10}$. The Bi-Maxwellian distribution is given by ${ }^{20}$

$$
\begin{aligned}
\mathscr{F}_{h}(s, \varepsilon, \mu)= & \mathscr{N}(s)\left(\frac{m_{h}}{2 \pi T_{\perp}(s)}\right)^{3 / 2} \\
& \times \exp \left[-m_{h}\left(\frac{\mu B_{C}}{T_{\perp}(s)}+\frac{\left|\varepsilon-\mu B_{C}\right|}{T_{||(s)}}\right)\right],
\end{aligned}
$$

where $\mathscr{N}(s)$ is a density like amplitude factor, $m_{h}, \varepsilon$, and $\mu$ are a particle's mass, energy, and magnetic moment, respectively, $B_{C}$ is the critical magnetic field at the particle deposition layer, and $T_{\|}$and $T_{\perp}$ are the parallel and perpendicular temperatures, respectively. An analytic integral of this expression leads to the hot-particle pressure component of Eq. (11). The scale factor $H(s, B)$ in case of a Bi-Maxwellian distribution depends on $B_{C}$ as it differs for trapped and passing particles. In plasma regions of $B>B_{C}$ one has ${ }^{21}$

$$
H(s, B)=\frac{B / B_{C}}{1-\frac{T_{\perp}}{T_{\|}}\left(1-\frac{B}{B_{C}}\right)},
$$

in regions of $B<B_{C}$ one has ${ }^{21}$

$$
\begin{aligned}
H(s, B) & =\frac{B}{B_{C}} \\
& \times \frac{1+\frac{T_{\perp}}{T_{\|}}\left(1-\frac{B}{B_{C}}\right)-2\left(\frac{T_{\perp}}{T_{\|}}\right)^{5 / 2}\left(1-\frac{B}{B_{C}}\right)^{5 / 2}}{\left[1-\frac{T_{\perp}}{T_{\|}}\left(1-\frac{B}{B_{C}}\right)\right]\left[1+\frac{T_{\perp}}{T_{\|}}\left(1-\frac{B}{B_{C}}\right)\right]} .
\end{aligned}
$$

The value of $B_{C}$ determines the amount of trapped particles and thus influences the distribution function with none of the anisotropic particles being trapped if $B_{C}$ is smaller than the minimal magnetic field strength $B_{\min }$.

To set a certain Bi-Maxwellian distribution function, three input parameters $a_{t}, a_{h}(s)$, and $B_{C}$ are passed to ANIMEC. The ratio of perpendicular to parallel temperature is set by $a_{t}=T_{\perp} / T_{\|}$, 
Analysis of influences of pressure anisotropies on the 3D MHD equilibrium in LHD

$a_{h}(s)$ specifies the radial hot-particle pressure profile $p_{h}(s)$ using a polynomial of order 20 , and $B_{C}$ sets the critical magnetic field. In the following, $B_{C}$ is chosen such that a significant amount of trapped particles is present unless stated differently.

Whilst in the ANIMEC input a temperature ratio is specified, the plasmas are better characterized using their pressures. The anisotropies are quantified in the following by the ratio of the beta values assigned to the anisotropic pressure component perpendicular to the magnetic field, $\beta_{\perp}$, and the anisotropic component parallel to the magnetic field, $\beta_{\|}$,

$$
\beta_{\perp / \|}=\frac{\beta_{\perp}}{\beta_{\|}}
$$

Note that $a_{t}$ and $\beta_{\perp / \|}$ share two fix points $\left(a_{t}, \beta_{\perp / \|}\right)$ at $(0,0)$ and $(1,1)$ due to $p_{\perp}$ being zero in the first and equal to $p_{\|}$in the second case.

To assess plasma equilibria for different hot-particle profiles, three different profiles, sketched in Fig. (1), are evaluated. First, a radially constant profile

$$
p_{h}^{f}(s)=c_{0},
$$

where $c_{0}$ is a dimensionless constant, is analyzed to benchmark the influences of pressure anisotropies. In order to cover a realistic parameter range in $\beta_{e q}, c_{0}=3$ was chosen in this case. The peak value and radial integral of this profile are both three. The second profile used models an edge-peaked hot-particle deposition

$$
p_{h}^{e}(s)=c_{0}\left(1-s^{4}\right)^{2} \cdot s^{4}
$$

Such profiles can be expected for plasma scenarios in which primarily the edge region is heated. The maximum of the profile is scaled to unity by setting $c_{0}=27 / 4$, giving rise to a radial integral of 0.369 . The lower integral value causes the differences between scenarios of different $p_{0}$ to be smaller than in the flat profile case. The third profile evaluated mimics a power deposition at the plasma center

$$
p_{h}^{c}(s)=c_{0}\left(1-s^{2}\right)^{4}
$$

For this profile, in order that the maximum value equals unity, $c_{0}=1$ is set, leading to a radial integral of 0.406 , again, significantly smaller than the flat profile case but similar to the edgepeaked one. 
Analysis of influences of pressure anisotropies on the 3D MHD equilibrium in LHD

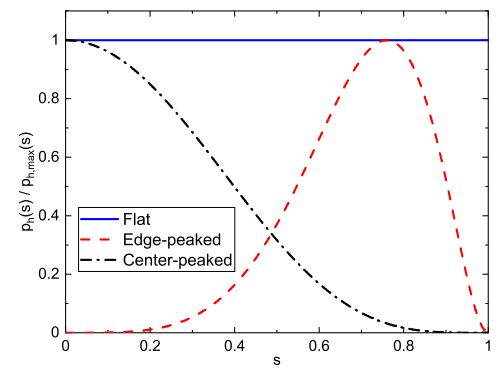

FIG. 1: Radial profiles of the investigated hot-particle pressure profiles. The coordinate $s$ is proportional to the enclosed toroidal flux with $s=\rho^{2}$.

\section{RESULTS}

The key parameter in the description of a 3D equilibrium is the plasmas magnetic axis position which changes due to the radial Shafranov-shift. This shift occurs due to a vertical magnetic field which again is caused by occurring Pfirsch-Schlüter currents. Besides the radial position of the magnetic axis, associated parameters such as the on-axis value of the rotational transform, $l$, or the vertical magnetic field, $B_{z}$, are of importance. As introduced in section II, anisotropic 3D equilibria may be assessed by $\beta_{t o t}$ or $\beta_{e q}$ with theory predicting that, when the magnetic axis position is plotted against $\beta_{e q}$, the anisotropic data points coincide with ones of isotropic plasmas of same $\beta$. Note that this match of data points may only be expected when the plasma can be described by the low beta ordering that the introduced theory is based on.

The framework used in this study is given by the LHD standard configuration whose magnetic saddle structure at an upright and recumbent plasma location is displayed in Fig. (2). The minimal magnetic field strengths in the two cross sections are $1.73 \mathrm{~T}$ and $1.74 \mathrm{~T}$, respectively, the maximal field strengths are $4.02 \mathrm{~T}$ and $3.28 \mathrm{~T}$, respectively.

The three scenarios introduced in III are assessed using an underlying parabolic thermal pressure profile $m(s)=m_{0}(1-s)$, where $s=\rho^{2}$. The ratio of the specific heats $\Gamma$ in Eqs. (9) and (10) is set to zero which relates to the incompressible plasma case in which the plasma mass $m_{0}$ reduces to the plasma pressure $p_{0} . p_{0}$ is varied to $1.0,1.5,2.0,2.5,3.0 \times 10^{4} \mathrm{~Pa}$ for each of the profiles. The number of radial grid points is 201 , the reference location of the magnetic axis position is obtained by setting $p_{0}=0 \mathrm{~Pa}$ and is found to $r_{0}=3.653 \mathrm{~m}$. A radial shift of the magnetic axis is 
Analysis of influences of pressure anisotropies on the 3D MHD equilibrium in LHD

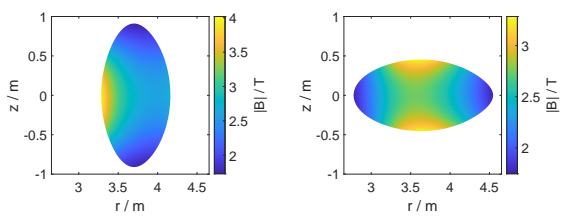

FIG. 2: Absolute vacuum magnetic field strength in the LHD standard configuration at the upright (left) and recumbent (right) locations of the plasma. The two locations are half a field period apart from another, therefore cover the whole field geometry, and relate to the most elongated cross sections in the respective directions.

defined as $\Delta R_{a x}=r\left(\beta_{\perp / \|}\right)-r_{0}$.

To assess parallel as well as perpendicular dominated anisotropic plasmas, the parameter range of $a_{t}=T_{\perp} / T_{\|}$is chosen to $[0,10]$, which also contains the two aforementioned fix points. The $a_{t}$ increments in the lower parameter region are chosen smaller than in the higher one due to a steeper scaling of $\beta_{\perp / \|}$ with $a_{t}$ for small values of $a_{t}$.

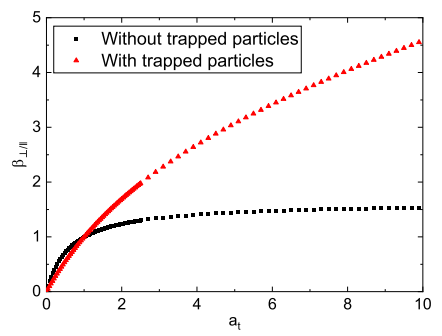

FIG. 3: Anisotropies reached in case of a flat hot-particle profile with $\left(B_{C}=2.125 \mathrm{~T}\right)$ and without $\left(B_{C}=1 \mathrm{~T}\right)$ trapped particles. The two fix points of $a_{t}$ and $\beta_{\perp / \|}$ are fulfilled in both cases, the different increments are chosen due to the scaling of $\beta_{\perp / \|}$ with $a_{t}$.

Examples for the relation of $a_{t}$ and $\beta_{\perp / \|}$ for one case with and one without trapped particles over the assessed parameter range are displayed in Fig. (3). The non-linear scaling of the two parameters as well as the necessity for smaller increments for low $a_{t}$ is apparent. The figure is revisited and discussed in depth in section IV A. 
Analysis of influences of pressure anisotropies on the 3D MHD equilibrium in LHD

\section{A. Flat profile case}

Fig. (4) depicts the radial location of the magnetic axis versus the two introduced beta definitions without trapped particles, obtained by setting $B_{C}=1 \mathrm{~T}$, a value significantly smaller than $B_{\min }$ of $1.73 \mathrm{~T}$. Colors ranging from red to pink indicate an increase of the pressure scale factor $p_{0}$ in increments of $0.5 \times 10^{4} \mathrm{~Pa}$. The black data points relate to magnetic axis locations obtained for isotropic plasmas with same $\beta$, using the same isotropic pressure profile. In compliance with the results obtained by Asahi et al. ${ }^{9}$ and Eq. (3), the magnetic axis location is a double-valued function when plotted against $\beta_{t o t}$ whilst it follows the approximately linear isotropic reference data when plotted against $\beta_{e q}$ as derived in section II. The deviations of the data points from the isotropic ones in Fig. (4a) are caused by the fact that the relation between the Shafranov-shift and the plasma energy is different for isotropic and anisotropic plasma components, consequently giving rise to a deviation from the isotropic reference data.

As indicated by the labeled data points in Fig. (4b), stronger perpendicular anisotropies, i.e. cases with high $a_{t}$, are found to have lower $\Delta R_{a x}$ with lower $\beta_{e q}$ values. These data points also lie closer together in the parameter space than the more sparse parallel dominated data points of low $a_{t}$ due to the saturation observed in Fig. (3).

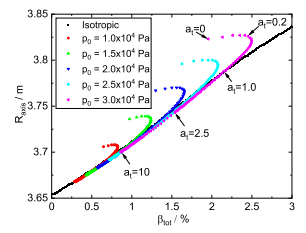

(a) Magnetic axis
position versus $\beta_{t o t}$.

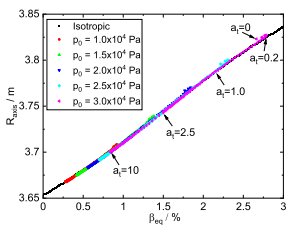

(b) Magnetic axis

position versus $\beta_{e q}$.

FIG. 4: Magnetic axis position versus the two beta definitions for different pressure scale factors $p_{0}$, in case of a flat hot-particle pressure profile and no trapped particles. Five pink data points are example wise labelled with their $a_{t}$ value. The location of these points for the other cases is alike. The isotropic reference case is given by the black (square-shaped) data points and shows a match with the anisotropic data when plotted against $\beta_{e q}$.

Introducing a population of trapped particles by increasing $B_{C}$ to $2.125 \mathrm{~T}$ leads to the magnetic axis positions displayed in Fig. (5). Fig. (5a) shows the magnetic axis position for the different 
Analysis of influences of pressure anisotropies on the 3D MHD equilibrium in LHD

$p_{0}$ versus $\beta_{e q}$ while Fig. (5b) shows a $\beta_{\perp / \|}$ resolved zoom onto the turquoise (diamond-shaped), $2.5 \times 10^{4} \mathrm{~Pa}$, data points in Fig. (5a).

Parallel dominated anisotropies are observed to lie on the line outlined by the isotropic reference data whilst strong perpendicular anisotropic cases show smaller $\Delta R_{a x}$ than the isotropic reference. The close up onto the data shows that deviations from the theoretical predictions start to occur when $\beta_{\perp / \|} \geq 2.0$. This behaviour is present regardless of the $p_{0}$ imposed, making it an effect inherent in the population of trapped particles and not a high pressure effect.
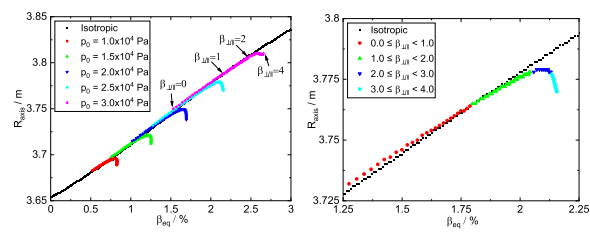
(a) Magnetic axis
(b) $\beta_{\perp / \|}$ resolved
locations for the
magnetic axis locations
different $p_{0}$ versus $\beta_{\text {eq }}$.
of the $2.5 \times 10^{4} \mathrm{~Pa}$
(turquoise/diamond-
shaped) data points in

(a) versus $\beta_{e q}$.

FIG. 5: Magnetic axis position versus $\beta_{e q}$ with a flat hot-particle pressure profile and trapped particles. The color code is chosen as before with four pink data points being example wise labelled with their respective $\beta_{\perp / \|}$ value. A match with the isotropic reference case is only present for low values of $\beta_{\perp / \|}$. $B_{C}$ was set to $2.125 \mathrm{~T}$.

The already introduced Fig. (3) displays the anisotropies reached in case of the data displayed in Figs. (4) and (5). While the simulations with trapped particles reach strong perpendicular anisotropies, for those without trapped particles $\beta_{\perp / \|}$ hardly exceeds 1.5 . This explains the differences between Figs. (4b) and (5a) as the perpendicular dominated parameter range necessary for the observed deviations to take place cannot be reached without trapped particles. The increase of $\beta_{\perp / \|}$ when introducing trapped particles is present as predominantly particles perpendicular to the magnetic field lines are trapped, significantly increasing their effects, subsequently increasing their pressure contribution and $\beta_{\perp / \|}$. Deviations from the isotropic reference data are therefore identified to occur for strong perpendicular anisotropies only which can only be reached in case of 
Analysis of influences of pressure anisotropies on the 3D MHD equilibrium in LHD a certain amount of trapped particles.

Fig. (6) depicts 2D pressure profiles of the parallel and perpendicular pressures $p_{\|}$and $p_{\perp}$ for the two LHD plasma locations of Fig. (2). The two locations were chosen such that they cover half a field period, allowing to assess the whole plasma due to symmetries in LHD. Note that the parallel and perpendicular profiles in the $\beta_{\perp / \|}=1.0$ case are equal due to the earlier mentioned fix point.
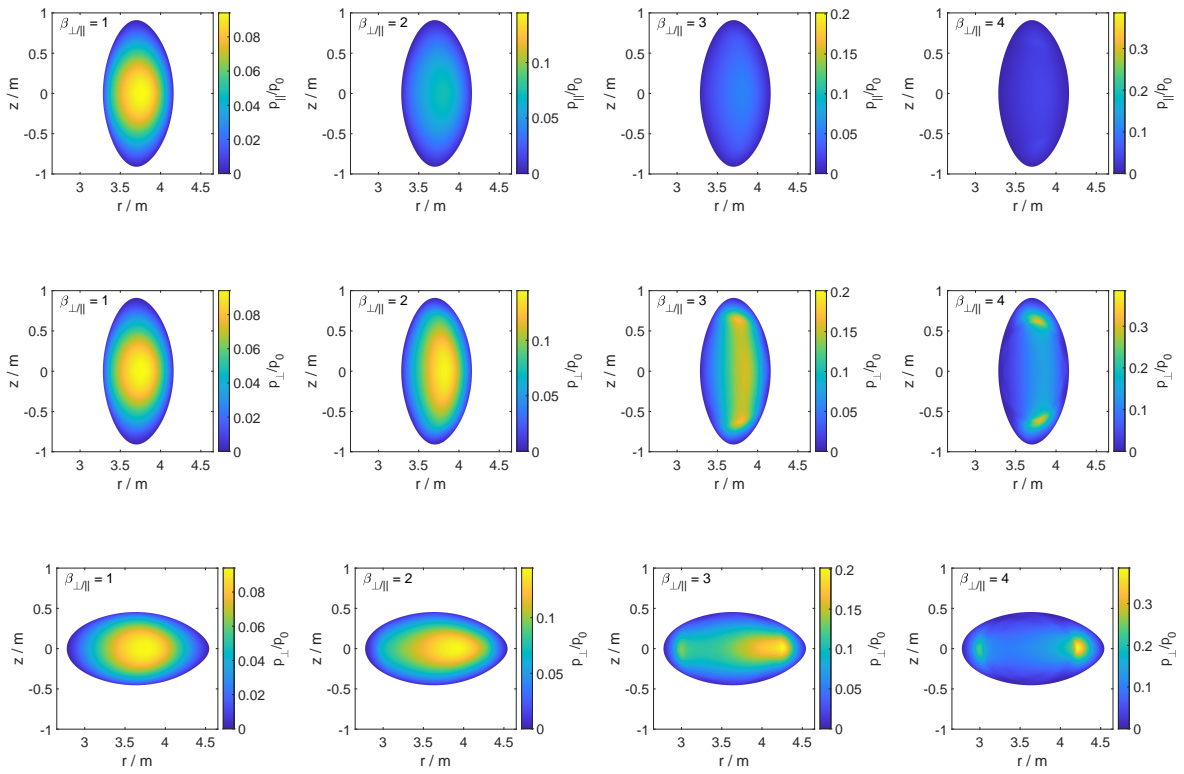

FIG. 6: Parallel (top one) and perpendicular (bottom two) pressure profiles at two LHD cross-sections for the flat hot-particle profile with trapped particles for $\beta_{\perp / \|}=1,2,3,4$ (left to right). The scaling is chosen such that the scaling of plots relating to one $\beta_{\perp / \|}$ is the same, $p_{0}$ is set to $2.5 \times 10^{4} \mathrm{~Pa}$.

The $p_{\|}$profiles show a decay in amplitude with increasing $\beta_{\perp / \|}$, and are almost vanished for $\beta_{\perp / \|}=4.0$, the $p_{\perp}$ profiles show a radial outward shift of the pressure peak up to $\beta_{\perp / \|}=2.0$ and a subsequent movement towards the plasma tips. The latter movement corresponds to a radial movement in case of the recumbent plasma cross section and an upward movement for the upright one. For large $\beta_{\perp / \|}$, localized pressure peaks are formed at the plasma tips at locations of minimal 
Analysis of influences of pressure anisotropies on the 3D MHD equilibrium in LHD

$|B|$. The location of the pressure peak for strong perpendicular anisotropies in the upright cross section matches density peaks observed by the vertical neutron camera system of LHD when the plasma is heated by perpendicular neutral beam injection ${ }^{22}$, indicating that the simulations cover a real physics mechanism and that the assessed parameter range can be experimentally reached in LHD. In Fig. (6) also note the symmetry in the upright cross section caused by the symmetric magnetic field in Fig. (2) and that the progression of the locations of the high $p_{\perp}$ regions is consistent with the path towards lowest $|B|$.

In analogy to positive pressure gradients giving rise to a radial outward Shafranov-shift, the inward trend of the magnetic axis location may be caused by the formation of negative pressure gradients. These are present as the pressure peaks are located at the tips of the plasma with the pressure falling off towards the plasma center, subsequently giving rise to negative pressure gradients in the direction of the magnetic axis. As positive pressure gradients give rise to an outward Shafranov-shift, these negative pressure gradients may be the cause for the negative trend of the magnetic axis location as it is present for $\beta_{\perp / \|}>2.0$ in Fig. (5).

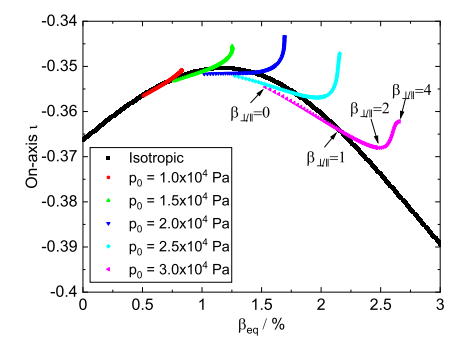

FIG. 7: Values of the rotational transform on the magnetic axis in case of a flat profile with trapped particles.

Besides the movement towards low $B$, Fig. (6) also shows that the anisotropic pressure components in LHD can no longer be treated as flux-surface constants for high $\beta_{\perp / \|}$ as local pressure maxima form. With the flux-surface-averaged treatment, which resulted from the low beta ordering used in the derivation of Eq. (2), being not applicable in such cases, the deviations from the isotropic reference observed in Fig. (5) can be explained.

As can be seen in the $\beta_{\perp / \|}=4.0$ plots of $p_{\perp}$ in Fig. (6) by a faint ellipse present at around $\rho=0.7$, the discretization of the ANIMEC grid starts to appear in the solutions, introducing nonphysical effects into the equilibria. As this behaviour is present regardless of the profile used, 
Analysis of influences of pressure anisotropies on the 3D MHD equilibrium in LHD

simulations with $\beta_{\perp / \|}>4.0$ are discarded in the following; this, together with convergence issues appearing for even larger $\beta_{\perp / \|}$, implies that the Bi-Maxwellian distribution function model implemented in ANIMEC can only treat perpendicular dominated anisotropies up to a certain level; no such problems are observed for parallel anisotropies. Note that these discretization problems persist when increasing the radial, poloidal, or toroidal node number.

Data for the on-axis $\imath$ value in case of the flat hot-particle profile with trapped particles in Fig. (7) shows an approximate match of the anisotropic and the isotropic data for $\beta_{\perp / \|} \leq 2.0$ and deviations for higher $\beta_{\perp / \|}$. In the fixed-boundary equilibrium framework, these changes are caused by changes of the total magnetic field at the location of the magnetic axis by means of currents in the plasma as described by Eq. (6), as well as by a radial movement of the magnetic axis within the total magnetic field. Which of the two effects is the dominant one can be assessed by looking at Fig. (5) which allows to gauge the strength of the latter effect by associating an anisotropic data point with an isotropic one of same radial location. Via the associated $\beta_{e q}$ values, these two points can in turn be identified in Fig. (7). In case that the deviations in the on-axis iota value are primarily caused by a movement of the magnetic axis within $\boldsymbol{B}_{t o t}$, the two data points are expected to have the same $l$ value. When checking for this behaviour in the given data points, the iota values do not match, indicating that the observed deviations are predominantly caused by a change of the total magnetic field rather than by a movement of the magnetic axis. Note that the observed changes in the on-axis $l$ value are small which is consistent with the expectation that any changes of $\boldsymbol{B}_{t o t}$ are in the order of $\beta$.

\section{B. Edge-peaked profile case}

The magnetic axis locations in case of the hot-particle profile specified in Eq. (18) and a critical magnetic field of 2.0 T are displayed in Fig. (8). The different $p_{0}$ values are color coded as in Fig. (4). A deviation from the isotropic reference and thus the theoretical scaling as it is expected for a classical heliotron is observed regardless of the $\beta_{e q}$ value. The data implies that, for an edge-peaked hot-particle profile, the magnetic axis barely moves.

Clarification over the small changes of the magnetic axis position is found in the $2 \mathrm{D}$ pressure plots of Fig. (9) which show that, caused by the imposed profile, only an ellipse determined by the shape of the plasma volume can be reached by the hot particles. With the pressure of the anisotropic particles being located at the edge of the plasma, negative pressure gradients in the 
Analysis of influences of pressure anisotropies on the 3D MHD equilibrium in LHD

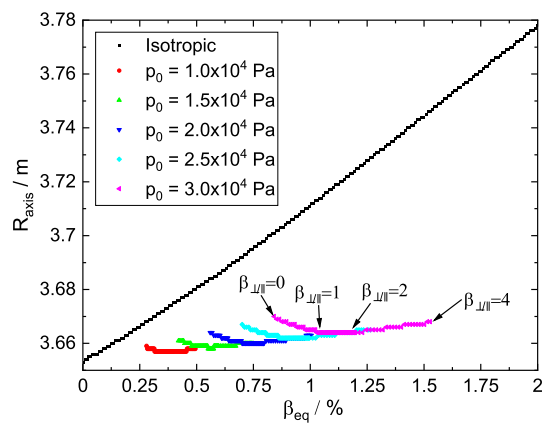

FIG. 8: Magnetic axis position versus $\beta_{e q}$ in case of an edge-peaked hot-particle pressure profile. $B_{C}$ was set to $2.0 \mathrm{~T}$.
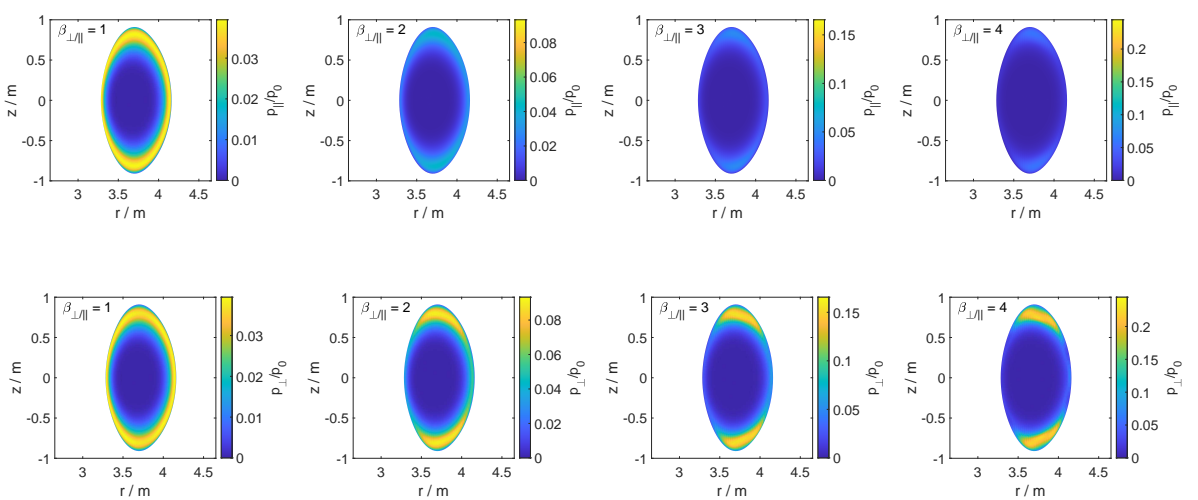

FIG. 9: Parallel (top) and perpendicular (bottom) pressure profiles for the edge-peaked pressure profiles for $\beta_{\perp / \|}=1,2,3,4$ (left to right) in case of the upright plasma cross section. $p_{0}$ is set to $2.5 \times 10^{4} \mathrm{~Pa}$.

direction of the magnetic axis are present. In analogy to the flat profile case, these gradients may reduce the outward Shafranov-shift, consequently giving rise to only small changes of the magnetic axis location as observed in Fig. (8). Note that, as these negative pressure gradients are present in the edge-peaked profile case regardless of $\beta_{\perp / \|}$, deviations from the isotropic reference data is seen throughout the whole parameter range. 
Analysis of influences of pressure anisotropies on the 3D MHD equilibrium in LHD

\section{Center-peaked profile case}

Center-peaked hot-particle pressure profiles lead to stronger shifts of the magnetic axis as in the isotropic reference case as apparent in Fig. (10).

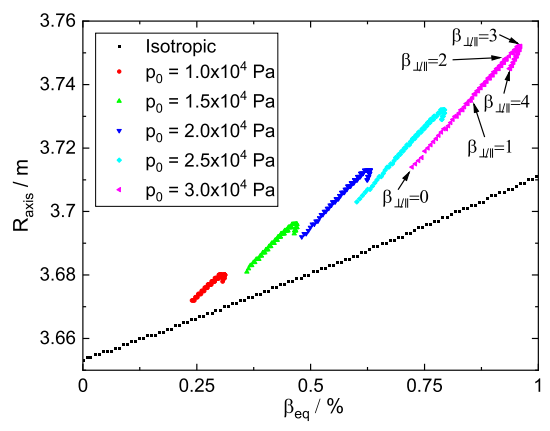

FIG. 10: Magnetic axis position versus $\beta_{e q}$ in case of a center-peaked hot-particle pressure profile. $B_{C}$ was set to $2.25 \mathrm{~T}$.
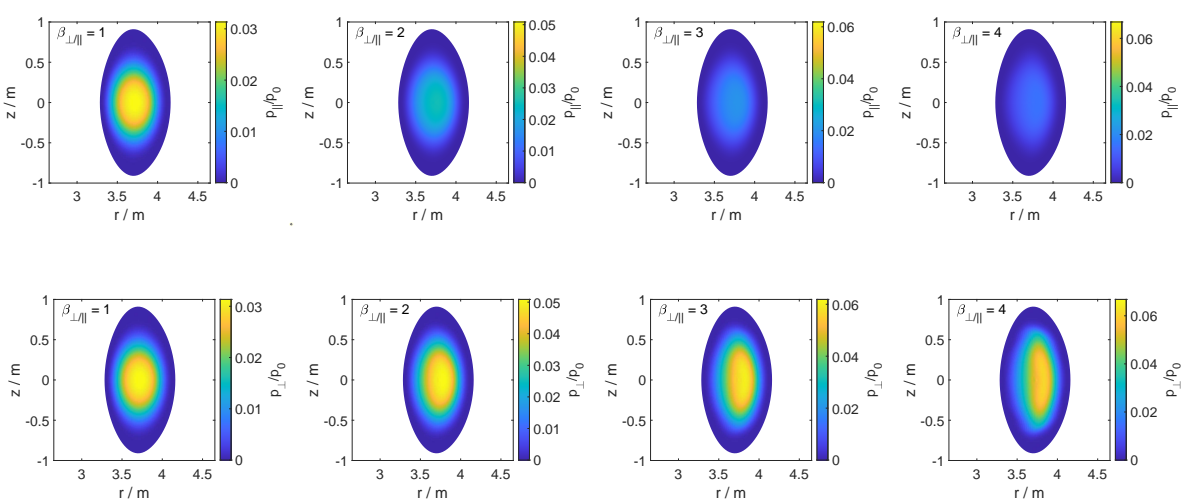

FIG. 11: Parallel (top) and perpendicular (bottom) pressure profiles for the center-peaked pressure profile for $\beta_{\perp / \|}=1,2,3,4$ (left to right) in case of the upright plasma cross section. $p_{0}$ is set to $2.5 \times 10^{4} \mathrm{~Pa}$.

Using the same color code as before, the different $p_{0}$ are indicated. An approximately linear but steeper scaling with $\beta_{e q}$ is found for $\beta_{\perp / \|} \leq 3$.0. Above this value, the magnetic axis position first 
Analysis of influences of pressure anisotropies on the 3D MHD equilibrium in LHD

stagnates and then, similar to the flat profile case, shrinks again. The $\beta_{e q}$ values are significantly smaller than in the other cases as the area accessible to the hot-particles is smaller and the set critical magnetic field strength of $2.25 \mathrm{~T}$ additionally reduces the peak pressures reached via $H(s, B)$. The $2 \mathrm{D}$ pressure plots in Fig. (11) show that the center-peaked case resembles a shrunk version of the flat profile case with the vertical elongation of the $p_{\perp}$ profile only taking place for $\beta_{\perp / \|}>3.0$. That this movement takes place for higher $\beta_{\perp / \|}$ values than in the flat profile case is caused by the fact that the magnetic field structure in the central area is different than a shrunk version of the whole plasma, giving rise to a different path towards minimal $|B|$, subsequently causing different $\beta_{\perp / \|}$ values to relate to different points on the path towards minimal $|B|$.

For $\beta_{\perp / \|}$ values larger than 3.0, $\Delta R_{a x}$ shrinks similar to the flat profile case. In analogy to the earlier discussed cases, this reduction of the Shafranov-shift may be caused by negative pressure gradients in the direction of the magnetic axis which form when the vertical elongation of the pressure maximum takes place. As the elongation takes place for larger $\beta_{\perp / \|}$ than in the other cases, an inward trend of the magnetic axis is only observed for stronger perpendicular anisotropies.

\section{Reassessment of the theory}

As introduced in section II, the framework of the treatment used by Hitchon et al. assumes a low beta ordering which reduces the pressure to its first order component, thus, making it constant on a given flux-surface. Based on Figs. (6), (9), and (11), this simplification was observed to be applicable in the LHD case for flat pressure anisotropies with $\beta_{\perp / \|} \leq 2.0$ only. The non-applicability of this ordering was found to match well with the deviations from the isotropic reference as observed in Figs. (5), (8), and (10), indicating that the observed deviations of the scaling of the magnetic axis location with $\beta_{e q}$ can be connected to a low beta ordering not being applicable in these cases.

To assess an additional link in the chain of reasoning towards the observed deviations, Fig. (12) depicts horizontal cuts of $\boldsymbol{B}_{z, p l}$ at $z=0$ in case of the different profiles for a low and a high $\beta_{\perp / \|}$ value at the upright plasma cross section. $\boldsymbol{B}_{z, p l}$ is determined based on Eq. (6) by means of subtracting the magnetic field in the vacuum case from the one in case of a given equilibrium. As the ANIMEC grid change with a movement of the magnetic axis position, $\boldsymbol{B}_{t o t}$ and $\boldsymbol{B}_{v a c}$ were approximated using linear splines which were then used for the subtraction. The rough trend around the indicated magnetic axis location is caused by the half-integer mesh of ANIMEC.

Regarding the validity of this approach, it should be noted that subtracting the vacuum mag- 
Analysis of influences of pressure anisotropies on the 3D MHD equilibrium in LHD

netic field configuration in a fixed-boundary framework does only give a defective version of the vertical magnetic field component originating from the plasma currents. Errors are introduced as the external field will react to a change of the plasma, subsequently moving the plasma boundary slightly. As such shifts are disregarded in a fixed-boundary framework, the field obtained by this approach only hints towards the real field component in the given case, allowing only a qualitative assessment of the underlying mechanism.

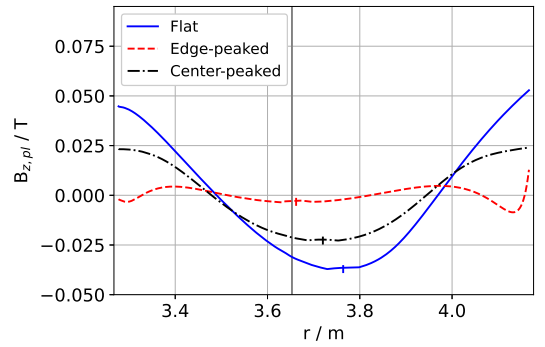

(a) Plasma current related component of the vertical magnetic field for $\beta_{\perp / \|}=1.0$.

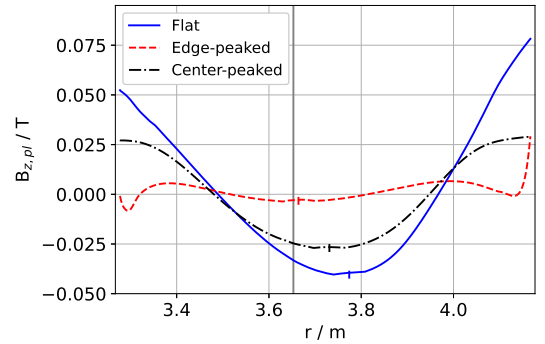

(b) Plasma current related component of the vertical magnetic field for $\beta_{\perp / \|}=3.5$.

FIG. 12: Horizontal cuts through $B_{z, p l}$ at $z=0$ for the assessed hot-particle profiles at the upright plasma location for $\beta_{\perp / \|}=1.0$ in (12a) and $\beta_{\perp / \|}=3.5$ in (12b). Vertical markers in the respective colors indicate the location of the magnetic axes in the respective cases, the gray vertical line relates to the vacuum location. Data was taken from the $p_{0}=2.5 \times 10^{4} \mathrm{~Pa}$ cases.

From Fig. (12), it is apparent that different radial profiles give rise to diverse profiles of $\boldsymbol{B}_{z, p l}$ whereby no direct connection between the pressure and $\boldsymbol{B}_{z, p l}$ profile is apparent.

For $\beta_{\perp / \|}=1.0$ in the flat profile case, $\boldsymbol{B}_{z, p l}$ is almost symmetric around the indicated magnetic axis. Such symmetries in $B_{z, p l}$ can be related to the pressure being a conserved quantity on flux-surfaces based on Eq. (5), which describes the Pfirsch-Schlüter current in the flux-surfaceaveraged case. From this equation, it follows that symmetric pressure gradients give rise to a symmetric Pfirsch-Schlüter current. As symmetric pressure gradients are caused by pressures which are constant on a given flux-surface, it follows that symmetric Pfirsch-Schlüter currents can be associated with flux-surface constant pressures. These symmetric currents in turn give rise to a symmetric magnetic field, therefore, allowing the association of a symmetric $B_{z, p l}$ with pressures being constants on flux-surfaces. The observed symmetry in $B_{z, p l}$ in the flat profile case therefore 
Analysis of influences of pressure anisotropies on the 3D MHD equilibrium in LHD

explains the match of the magnetic axis position with the isotropic reference for low $\beta_{\perp / \|}$ values as the underlying ordering is applicable.

In case of the center-peaked profile, the deviations from a mirror symmetry are even smaller, hinting towards data close to the isotropic reference. When looking at Fig. (10), it becomes apparent that, even though the radial shifts are stronger than in the isotropic reference case, the scaling with $\beta_{e q}$ nonetheless is similar to the one of the isotropic data. The right scaling with $\beta_{e q}$ can be assigned to a flux-surface-average treatment being valid while the stronger shifts can be assigned to the fact that only a part of the plasma is accessible by the hot particles, subsequently giving rise to a lower $\beta_{e q}$. These two effects together give rise to a large displacement of the magnetic axis at comparably low $\beta_{e q}$ as observed in Fig. (10).

In case of the edge-peaked profile, $\boldsymbol{B}_{z, p l}$ is almost zero around the magnetic axis and shows an asymmetry at the plasma edge, consistent with the imposed hot particle density profile. The asymmetry in turn hints towards the non-applicability of a low beta ordering for edge peaked hot-particle profiles, explaining the deviations observed in Fig. (8).

In case of the strong perpendicular anisotropies with $\beta_{\perp / \|}=3.5$ in Fig. (12b), the asymmetry around the magnetic axes in case of the flat profile becomes more distinct, hinting towards the non-applicability of the low beta ordering as was observed in Fig. (5) for strong perpendicular anisotropies. In the center-peaked profile case, the symmetry at the magnetic axis location remains almost intact, matching the observation that only small deviations from a rescaled isotropic reference occur as seen in Fig. (10). In the edge-peaked profile case, the asymmetry becomes even stronger but keeps its overall shape, indicating, as it was observed in the pressure plots of Fig. (9), the non-applicability of the low beta ordering, and subsequently explaining the deviations observed in Fig. (8).

\section{CONCLUSION}

Fixed-boundary plasma equilibria with tangential and perpendicular dominated pressure anisotropies with different hot-particle profiles in the LHD framework have been investigated. The hot-particles were modelled by a Bi-Maxwellian distribution function. The on-axis $l$ value is found to be primarily altered by a change of the total magnetic field due to plasma currents. The magnetic axis position in the flat profile case is found to follow theoretical predictions for a classical low-beta expansion heliotron model in case of parallel or weak perpendicular anisotropies with $\beta_{\perp / \|} \leq 2.0$, 
Analysis of influences of pressure anisotropies on the 3D MHD equilibrium in LHD

whereas strong perpendicular anisotropies lead to results deviating from the theoretically predicted scaling. The deviations are found to be caused by the non-applicability of a low beta ordering in such cases as the anisotropic pressure components exhibit significant higher order components. Simulated anisotropic pressure profiles are found to qualitatively match experimental density measurements in LHD plasmas heated by perpendicular neutral beam injection. As a potential mechanism underlying the observed inward movement of the magnetic axis, the formation of negative pressure gradients is proposed. Non-flat hot-particle profiles are found to deviate from theoretical predictions regardless of the anisotropy imposed with edge-peaked profiles holding the magnetic axis position fixed while center-peaked profiles give rise to a magnetic axis shift stronger than in the flat profile case.

With differences in runtime between ANIMEC and VMEC being small and certain scenarios showing differences in the magnetic axis location in the order of $10 \%-20 \%$ of the minor radius, it is advised to take into account the heating scenario in future investigations of 3D equilibria. Especially plasmas with radially localized heating or strong perpendicular anisotropies are expected to show deviations from the isotropic reference.

Subjects of future research are a new theoretical framework predicting anisotropies over a larger parameter range by taking into account higher order terms of the anisotropic pressure components as well as a systematical assessment of the influences of the magnetic field structure and the plasma geometry onto $\Delta R_{a x}$. In addition to the results obtained in case of LHD in this study, similar investigations of other stellarators, such as W7-X, may give an insight on how general the obtained findings are. Additionally, the role of pressure anisotropies in future reactors needs to be analysed systematically in order to estimate their influences onto the reactor design parameters. A systematic comparison of numerical results and experimental data needs to be performed in a next step.

In the context of ANIMEC itself, a comparison of the results with ones obtained using the modified slowing-down distribution is expected to give an insight into the limitations and areas of applicability of the two distribution functions.

\section{ACKNOWLEDGMENTS}

The authors greatly acknowledge instructive discussions with Dr. W.A. Cooper. One of the authors (Y.S.) received funding by "PLADyS", JSPS Core-to-Core Program A., Advanced Research 
Analysis of influences of pressure anisotropies on the 3D MHD equilibrium in LHD

Networks. The other authors (T.R. and J.P.) work has been carried out within the framework of the EUROfusion Consortium and has received funding from the Euratom research and training programme 2014-2018 and 2019-2020 under grant agreement No 633053. The views and opinions expressed herein do not necessarily reflect those of the European Commission.

The data that support the findings of this study are available from the corresponding author upon request.

\section{REFERENCES}

${ }^{1}$ K. Watanabe, S. Sakakibara, Y. Narushima, H. Funaba, K. Narihara, K. Tanaka, T. Yamaguchi, K. Toi, S. Ohdachi, O. Kaneko, H. Yamada, Y. Suzuki, W. Cooper, S. Murakami, N. Nakajima, I. Yamada, K. Kawahata, T. Tokuzawa, A. Komori, and LHD Experimental Group, Nuclear Fusion 45, 1247 (2005).

${ }^{2}$ T. Yamaguchi, K. Watanabe, S. Sakakibara, Y. Narushima, K. Narihara, T. Tokuzawa, K. Tanaka, I. Yamada, M. Osakabe, H. Yamada, K. Kawahata, K. Yamazaki, and LHD Experimental Group, Nuclear Fusion 45, L33 (2005).

${ }^{3}$ K. Watanabe, Y. Suzuki, S. Sakakibara, T. Yamaguchi, Y. Narushima, Y. Nakamura, K. Ida, N. Nakajima, H. Yamada, and LHD Experimental Group, Fusion Science and Technology 58, 160 (2010).

${ }^{4}$ S. Hirshman and O. Betancourt, Journal of Computational Physics 96, 99 (1991).

${ }^{5}$ W. A. Cooper, S. P. Hirshman, P. Merkel, J. P. Graves, J. Kisslinger, H. F. Wobig, Y. Narushima, S. Okamura, and K. Y. Watanabe, Computer Physics Communications 180, 1524 (2009).

${ }^{6}$ W. Cooper, J. Graves, S. Hirshman, T. Yamaguchi, Y. Narushima, S. Okamura, S. Sakakibara, C. Suzuki, K. Watanabe, H. Yamada, and K. Yamazaki, Nuclear fusion 46, 683 (2006).

${ }^{7}$ W. Cooper, S. Hirshman, S. Merazzi, and R. Gruber, Computer physics communications 72, 1 (1992).

${ }^{8}$ W. Cooper, S. Hirshman, T. Yamaguchi, Y. Narushima, S. Okamura, S. Sakakibara, C. Suzuki, K. Watanabe, H. Yamada, and K. Yamazaki, Plasma physics and controlled fusion 47, 561 (2005).

${ }^{9}$ Y. Asahi, Y. Suzuki, K. Watanabe, and W. A. Cooper, Plasma and Fusion Research 6, 2403123 (2011).

${ }^{10}$ J. Graves, W. Cooper, S. Coda, L.-G. Eriksson, T. Johnson, and J.-E. Contributors (American 
Analysis of influences of pressure anisotropies on the 3D MHD equilibrium in LHD Institute of Physics, 2006) p. 350.

${ }^{11}$ W. Cooper, Y. Asahi, Y. Narushima, Y. Suzuki, K. Watanabe, J. Graves, and M. Y. Isaev, Physics of Plasmas 19, 102503 (2012).

${ }^{12}$ W. Hitchon, Nuclear Fusion 23, 383 (1983).

${ }^{13}$ W. Hitchon and P. Fielding, Nuclear Fusion 21, 775 (1981).

${ }^{14}$ J. Greene and J. Johnson, The Physics of Fluids 4, 875 (1961).

${ }^{15}$ G. Chew, M. Goldberger, and F. Low, Proceedings of the Royal Society of London. Series A. Mathematical and Physical Sciences 236, 112 (1956).

${ }^{16}$ V. Pustovitov, Plasma Physics and Controlled Fusion 52, 065001 (2010).

${ }^{17}$ P. Fielding and W. Hitchon, Journal of Plasma Physics 24, 453 (1980).

${ }^{18}$ H. Grad, The Physics of Fluids 9, 498 (1966).

${ }^{19}$ S. Hirshman, W. van Rij, and P. Merkel, Computer Physics Communications 43, 143 (1986).

${ }^{20}$ K. McClements, R. Dendy, R. Hastie, and T. Martin, Physics of Plasmas 3, 2994 (1996).

${ }^{21}$ W. Cooper, J. Graves, M. Jucker, K. Watanabe, Y. Narushima, and T. Yamaguchi, Plasma Physics and Controlled Fusion 49, 1177 (2007).

${ }^{22}$ K. Ogawa, M. Isobe, H. Kawase, T. Nishitani, R. Seki, M. Osakabe, and L. E. Group, Plasma Physics and Controlled Fusion 60, 044005 (2018). 


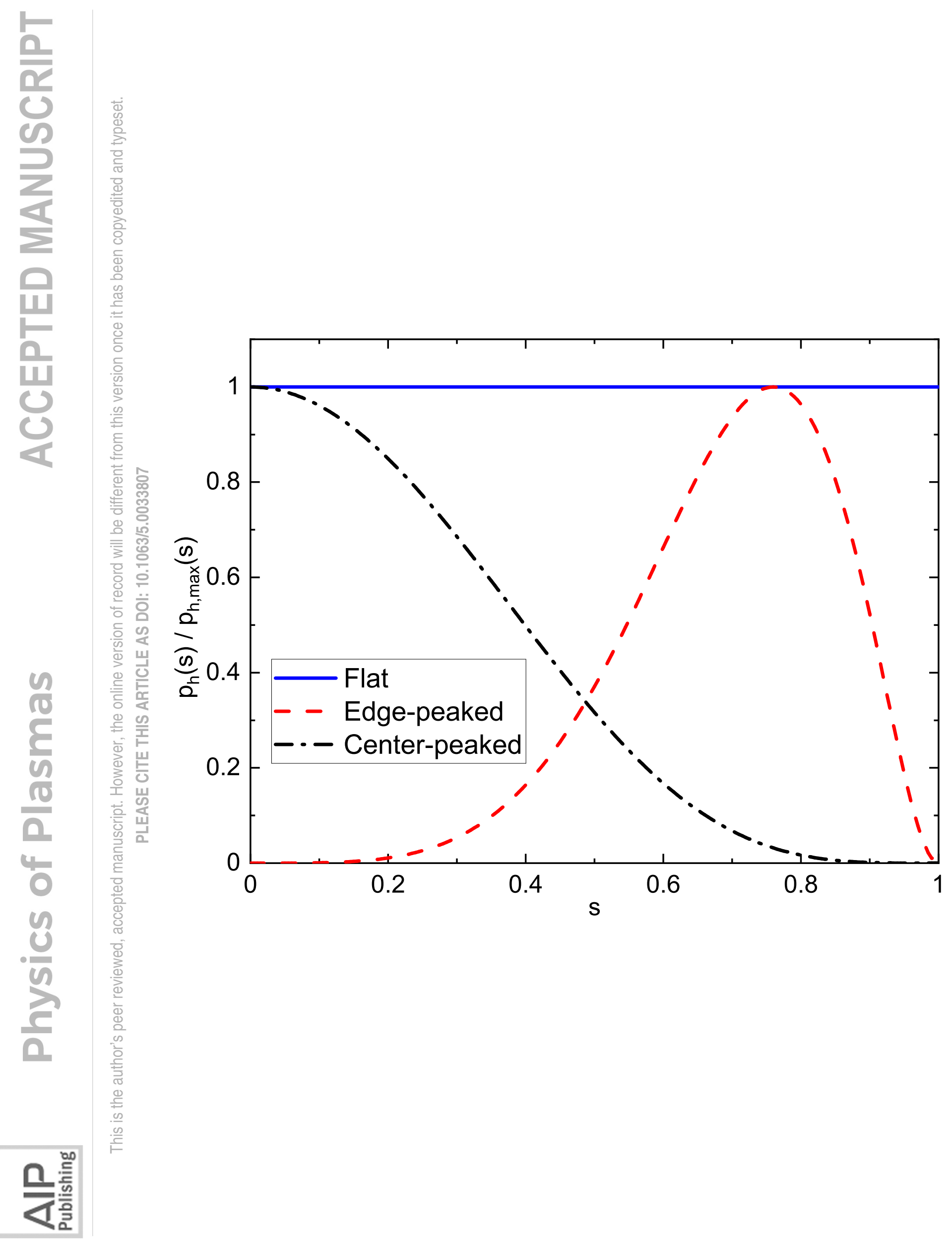




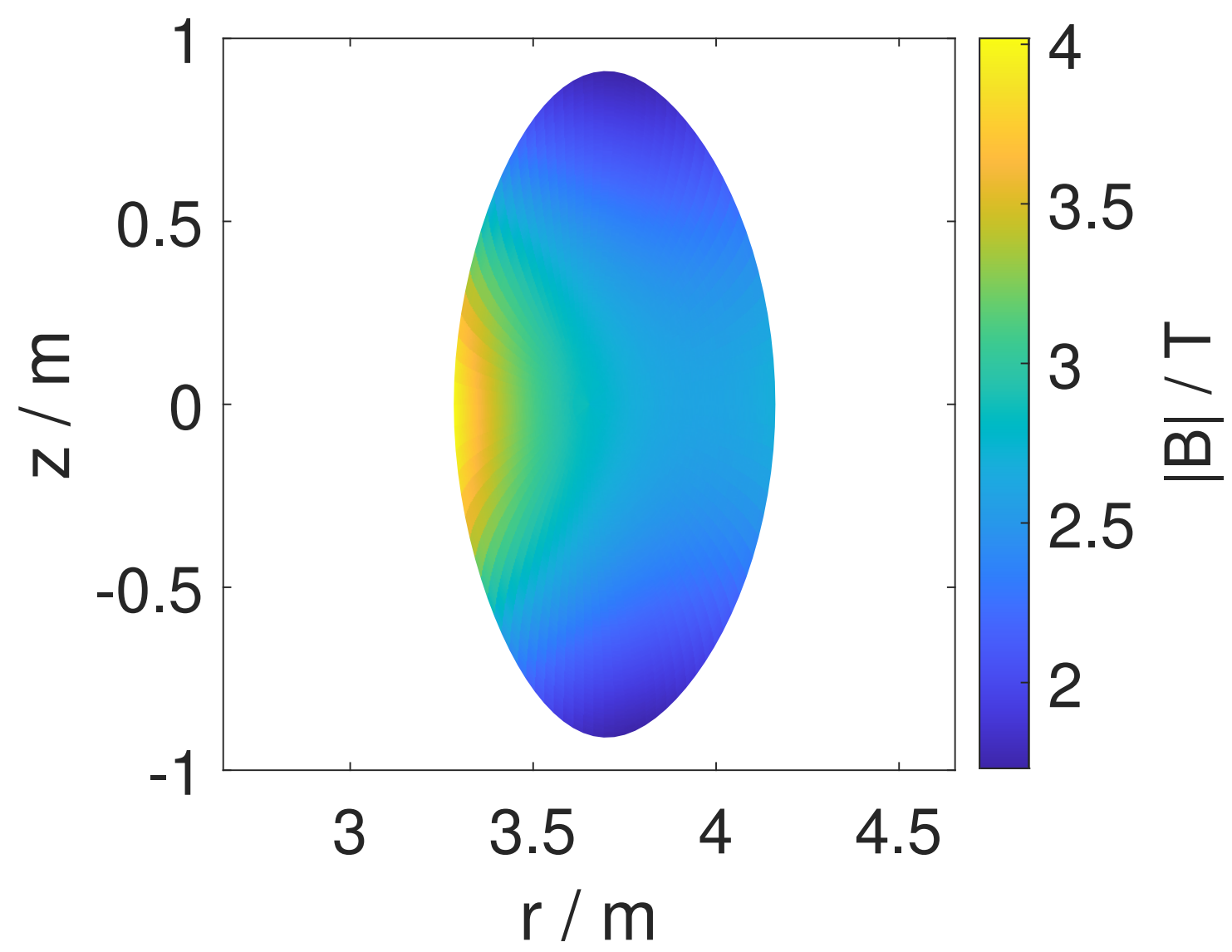

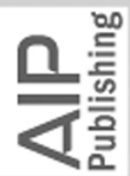




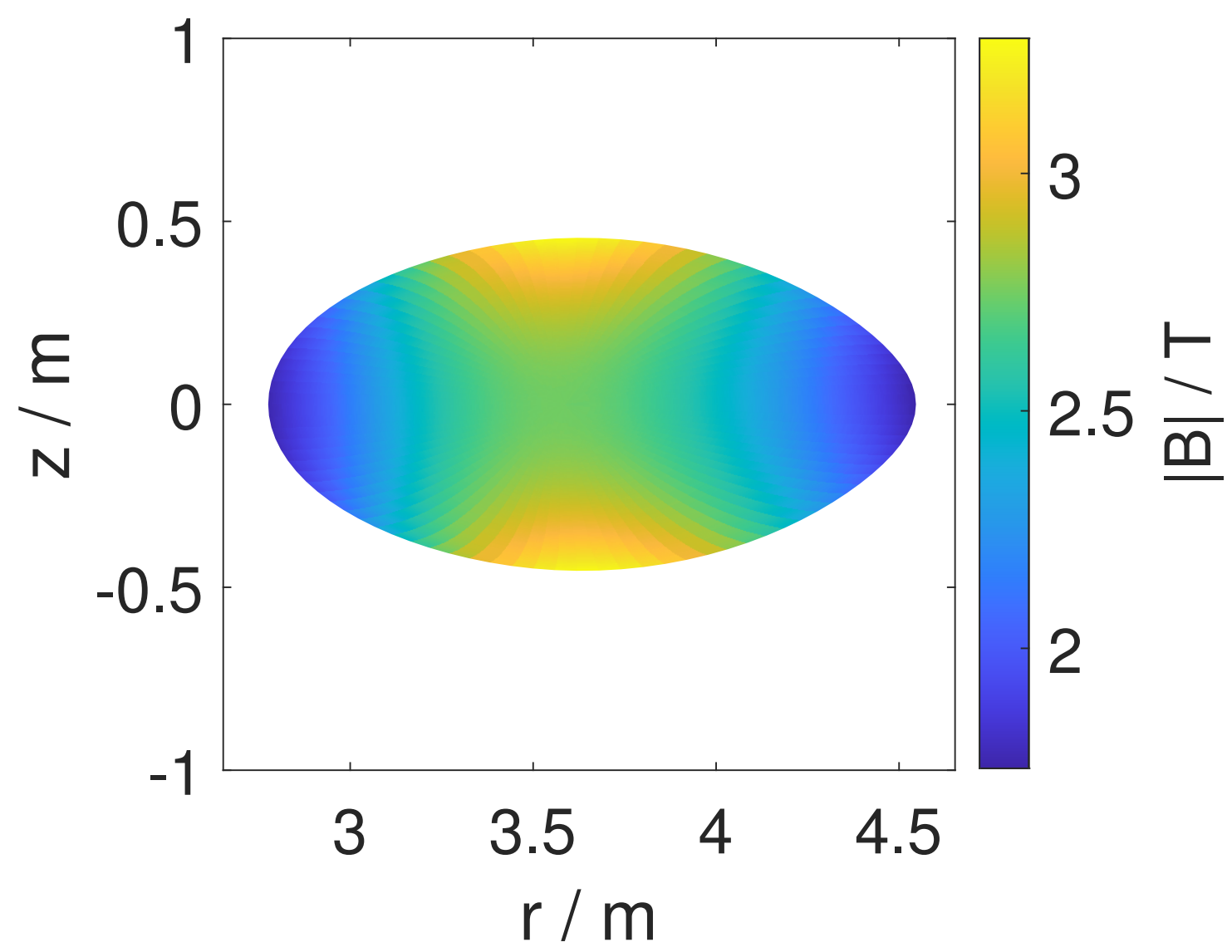

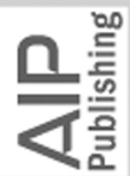




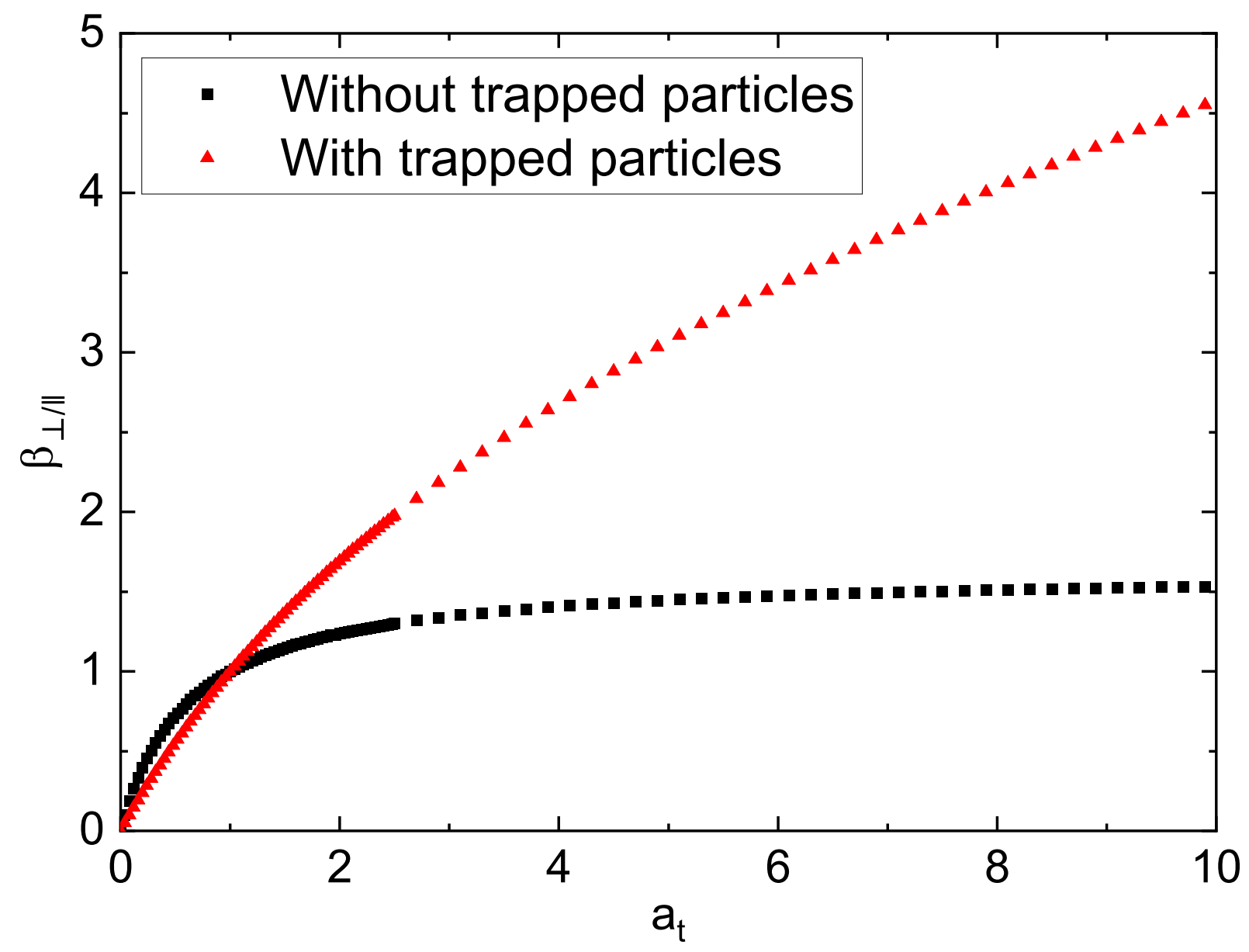




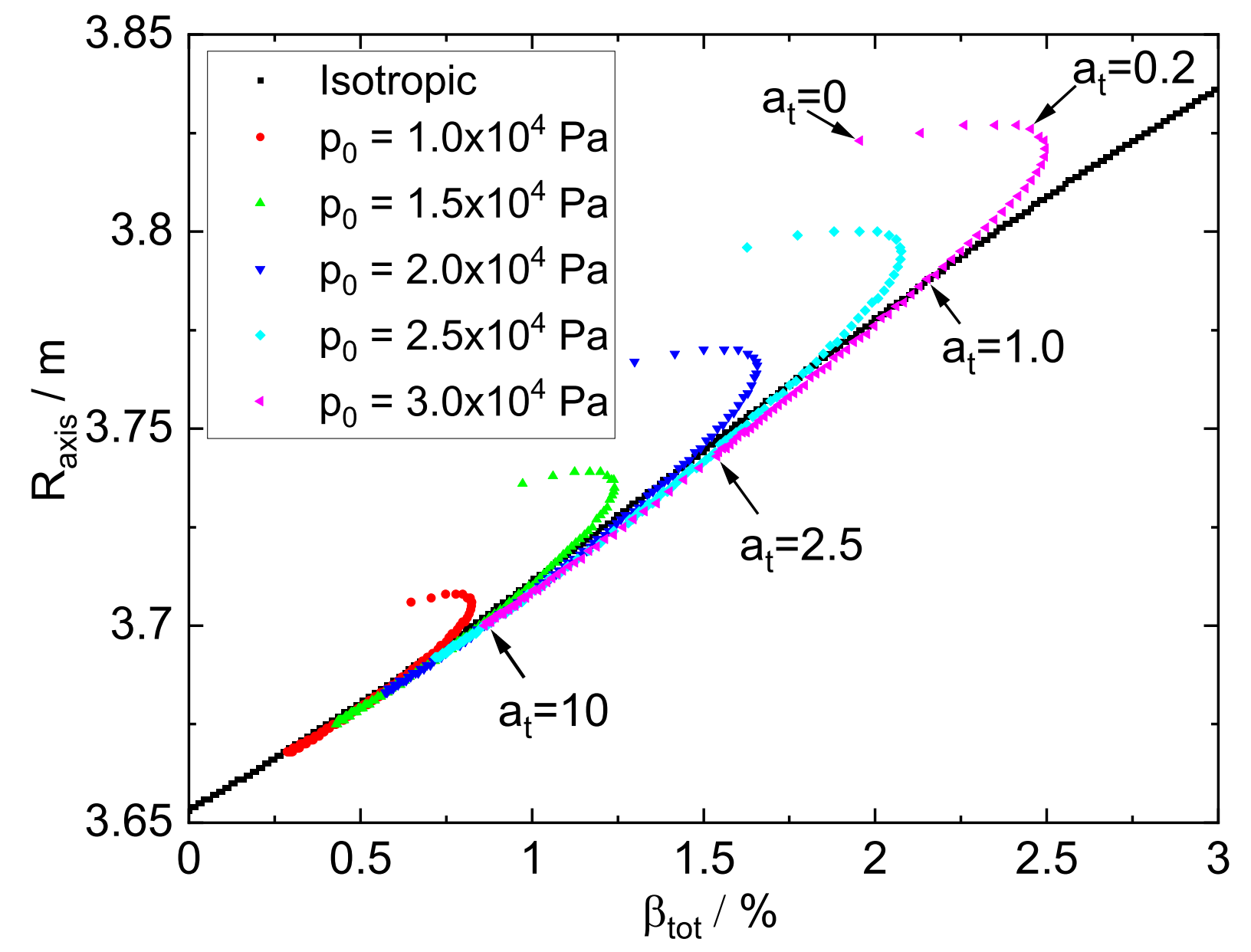




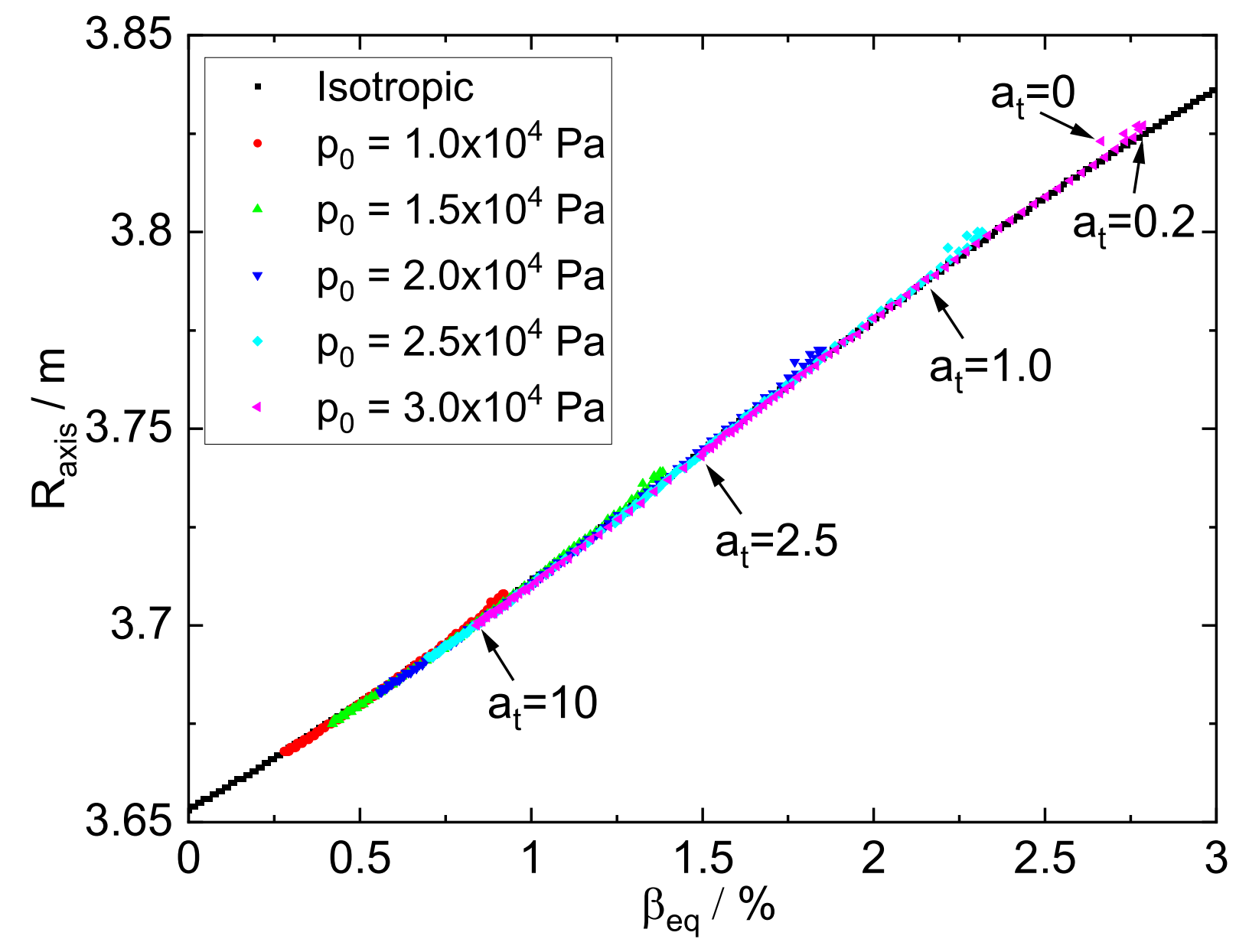




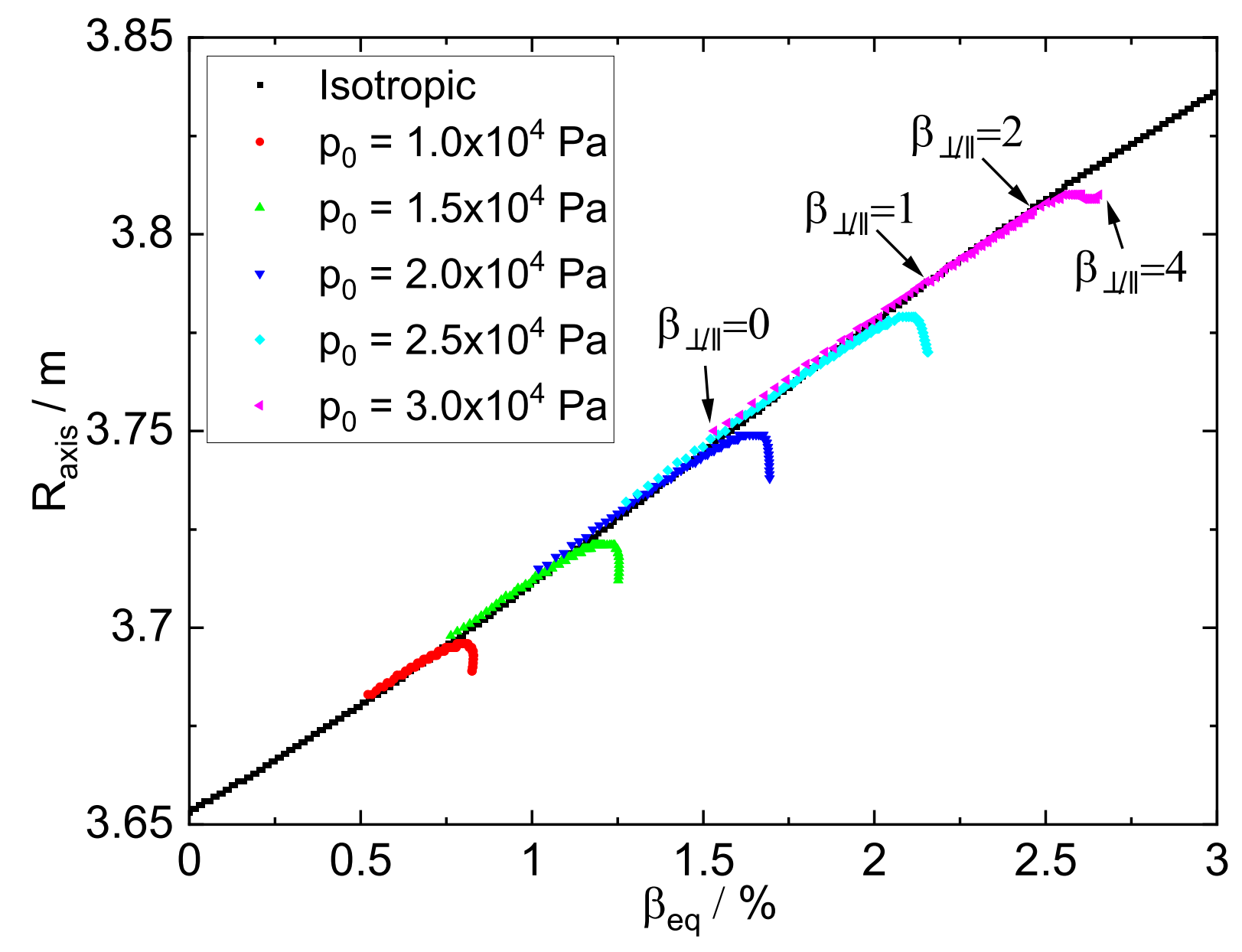




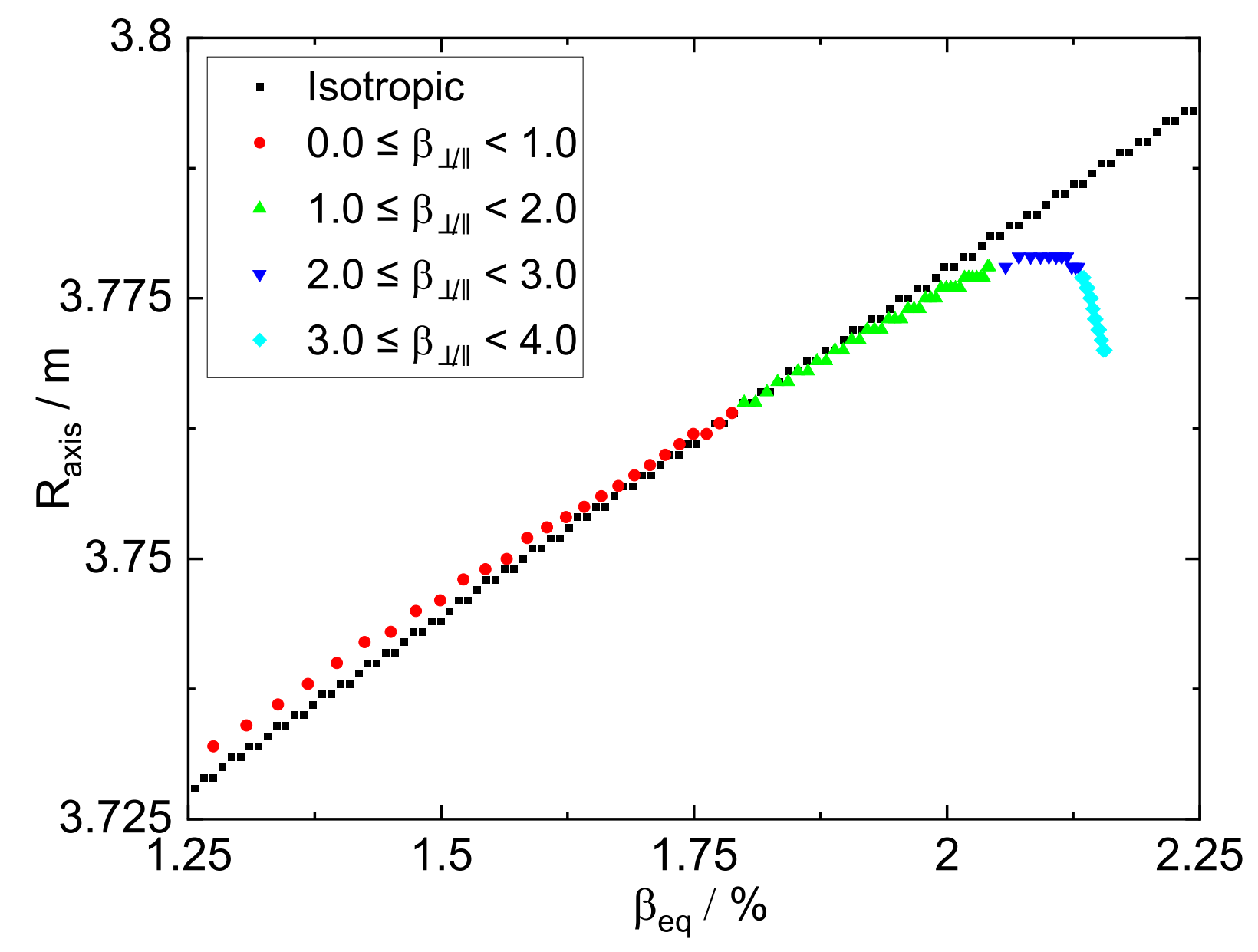




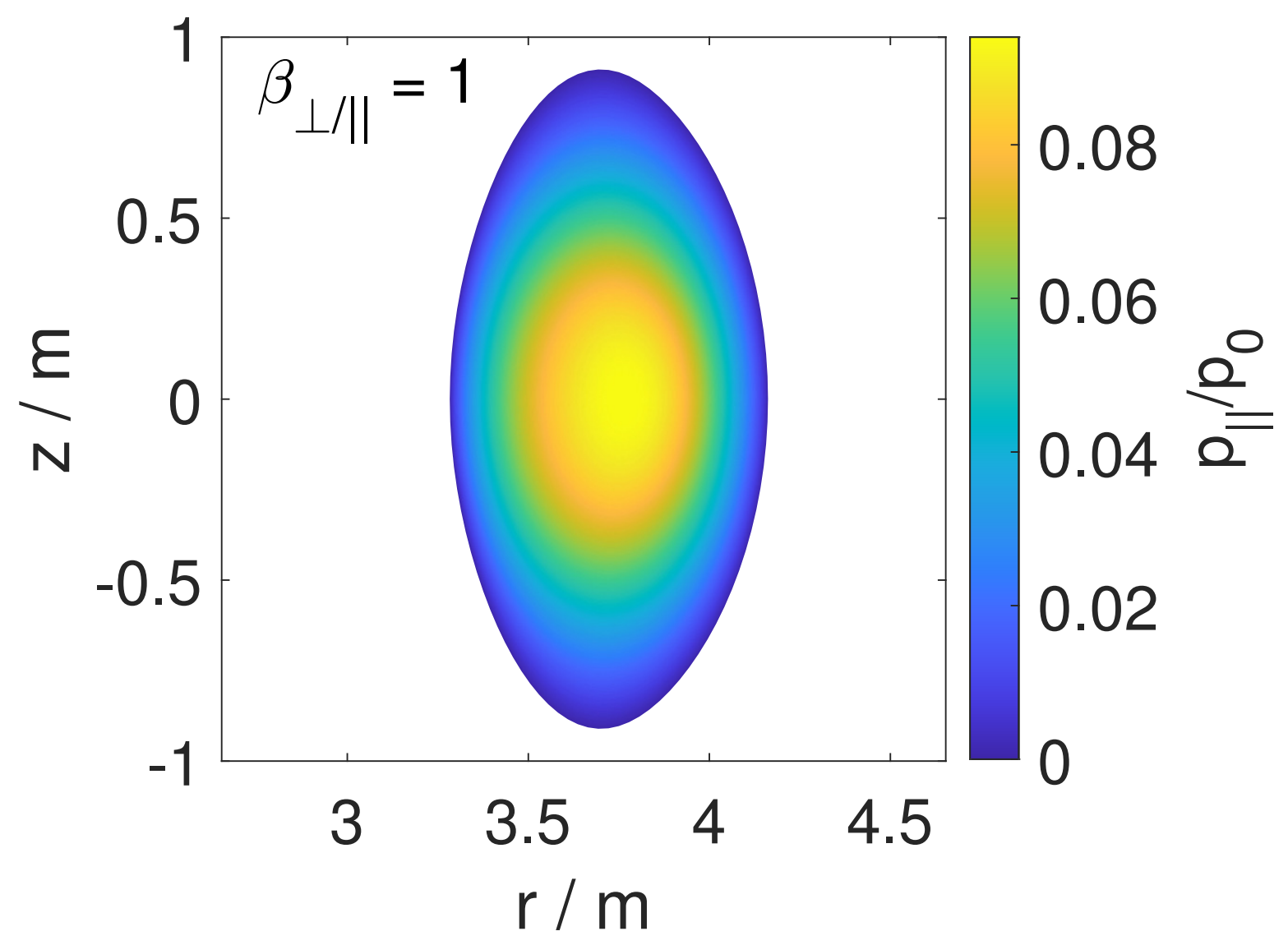

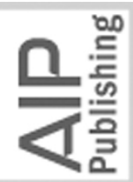




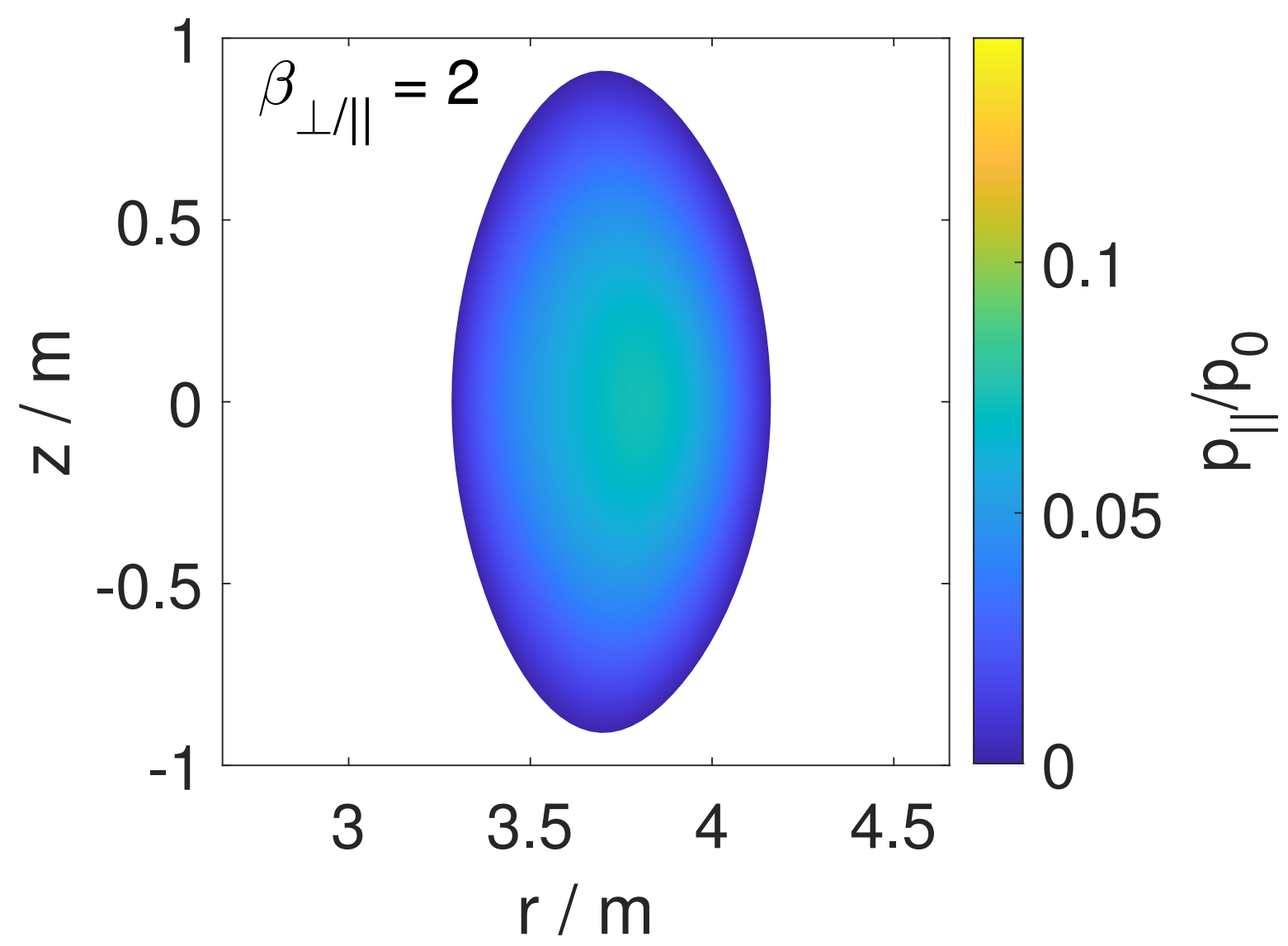

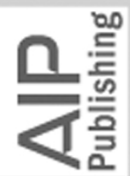




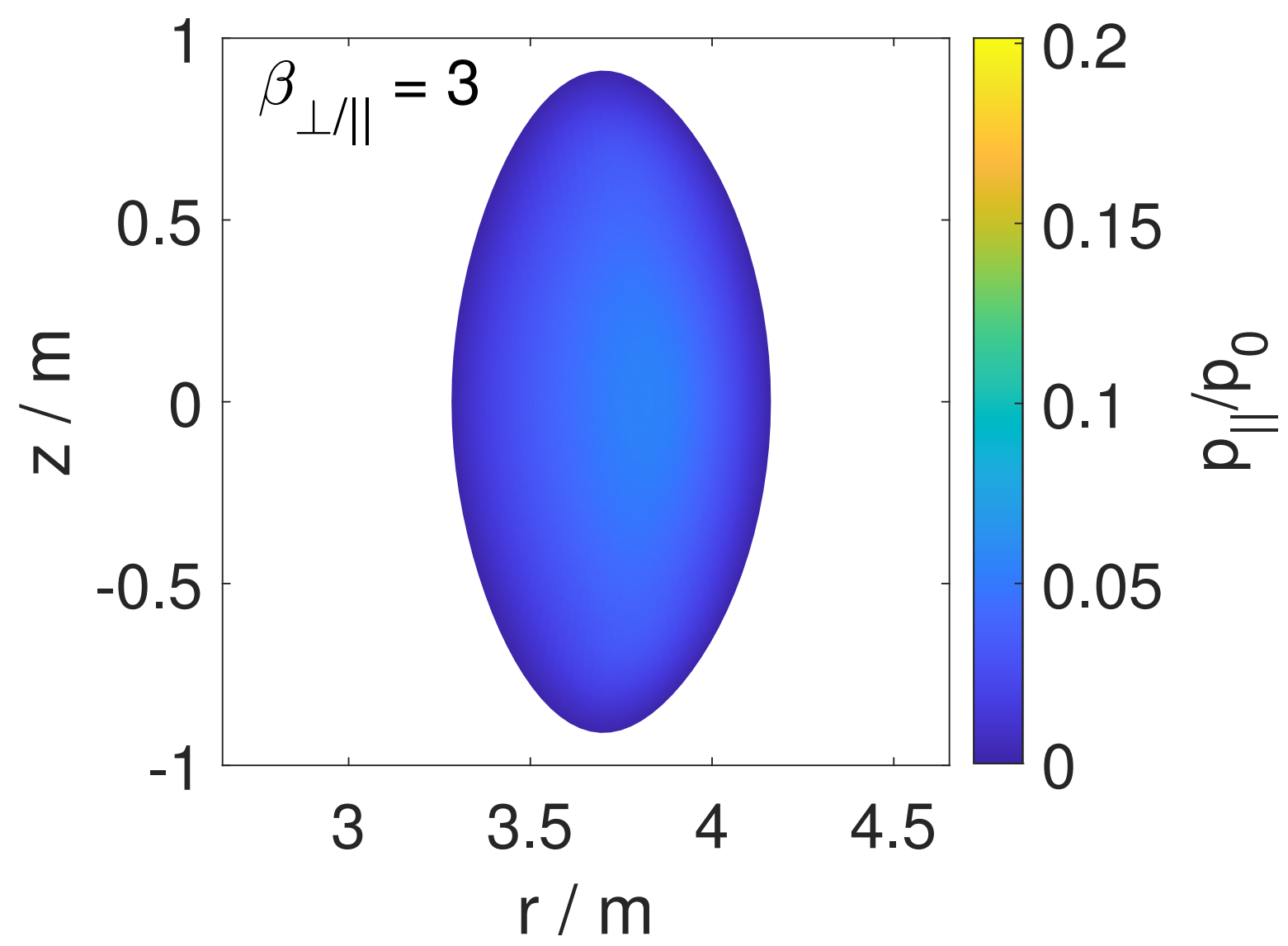

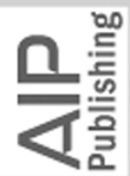




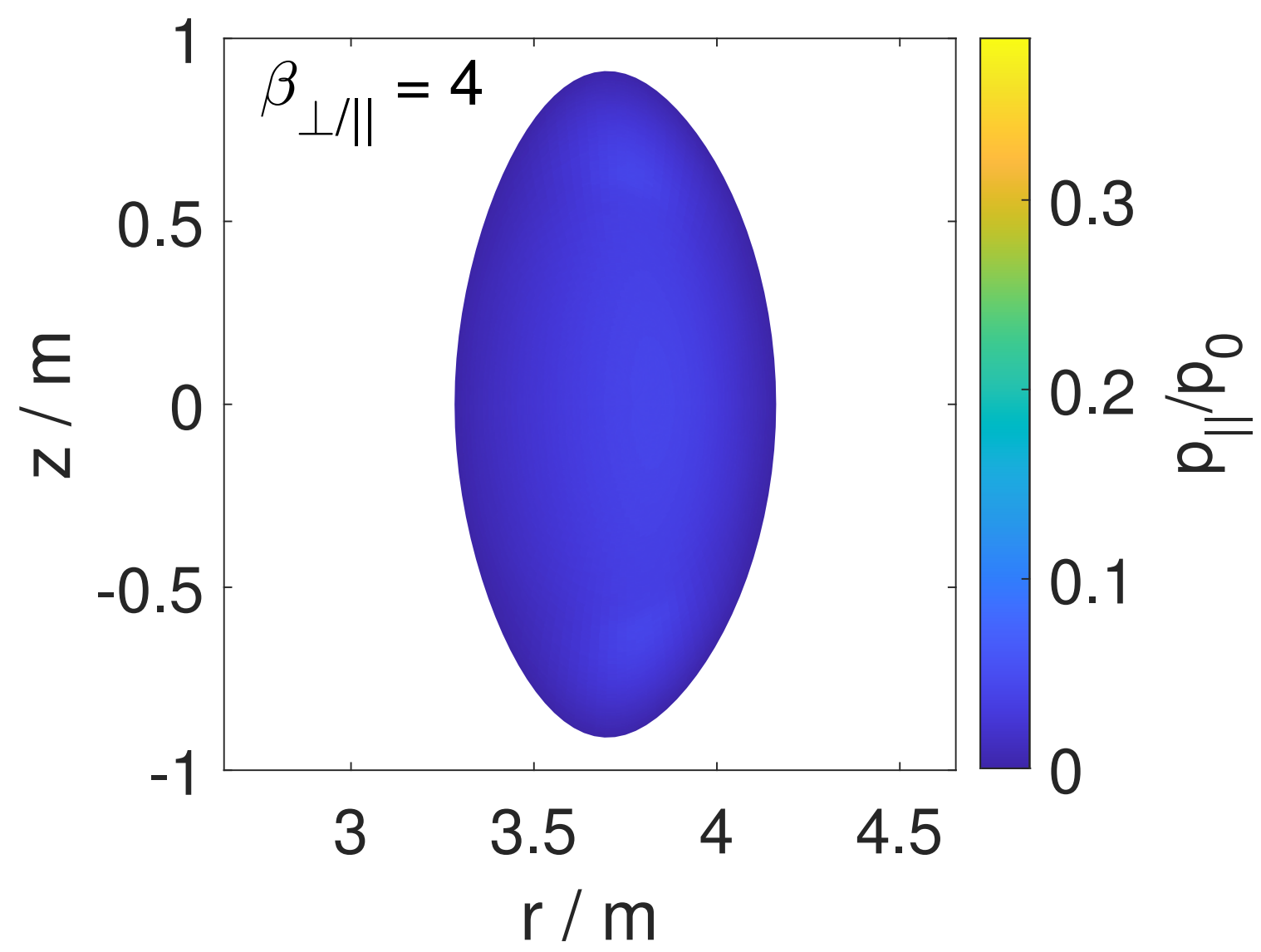

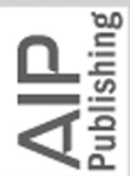




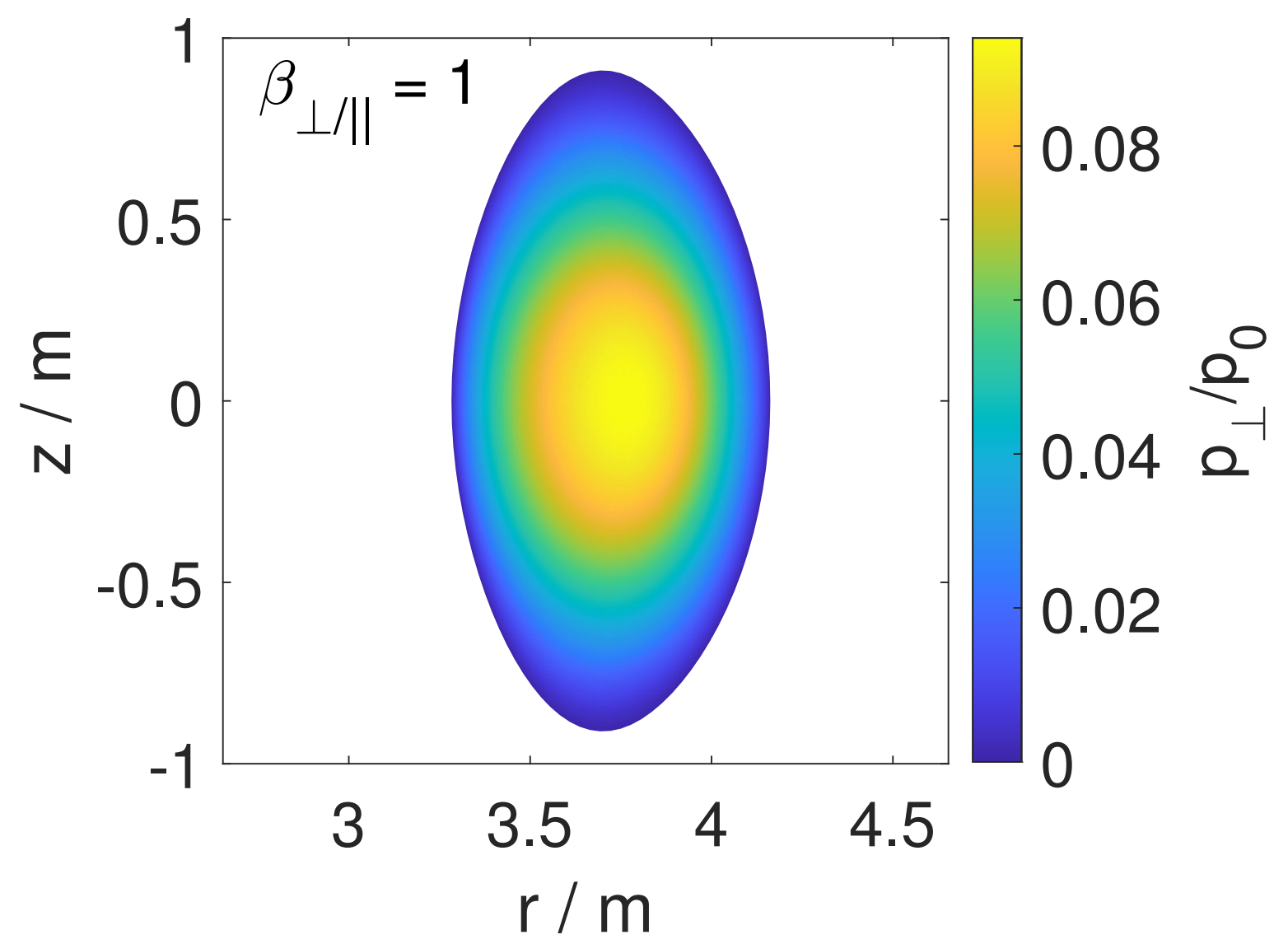

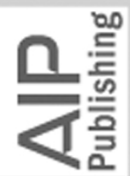




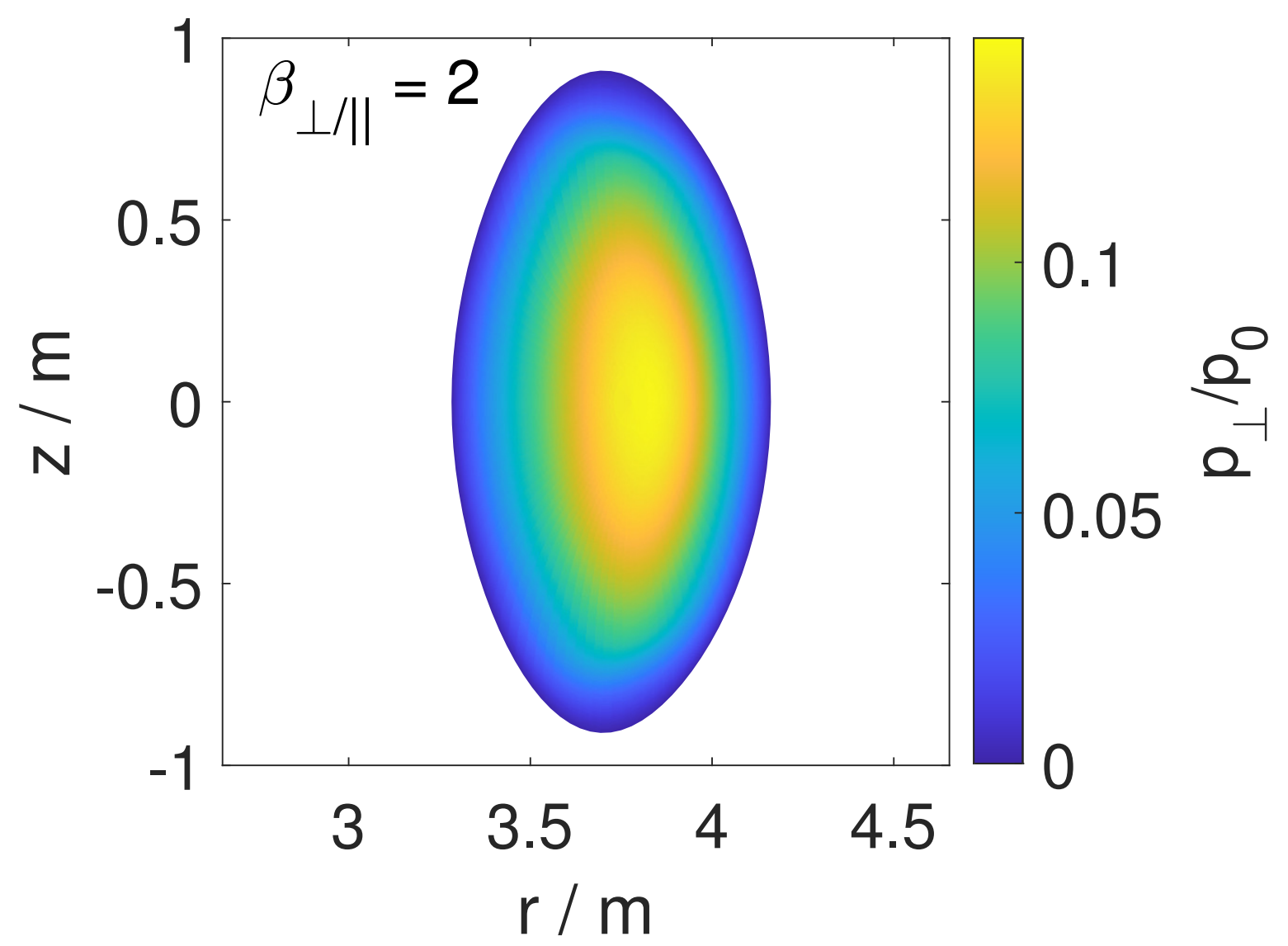

는 


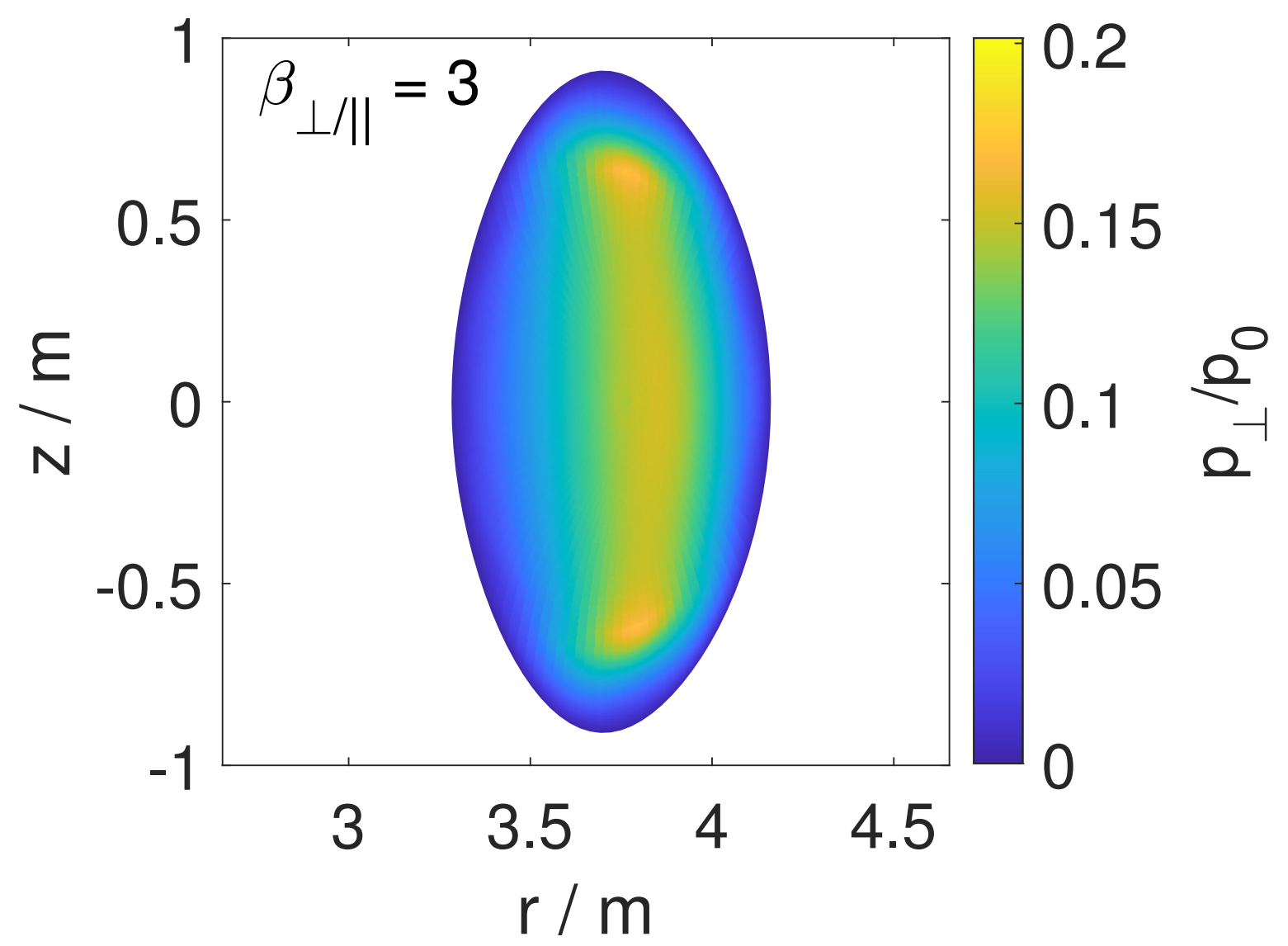

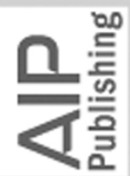




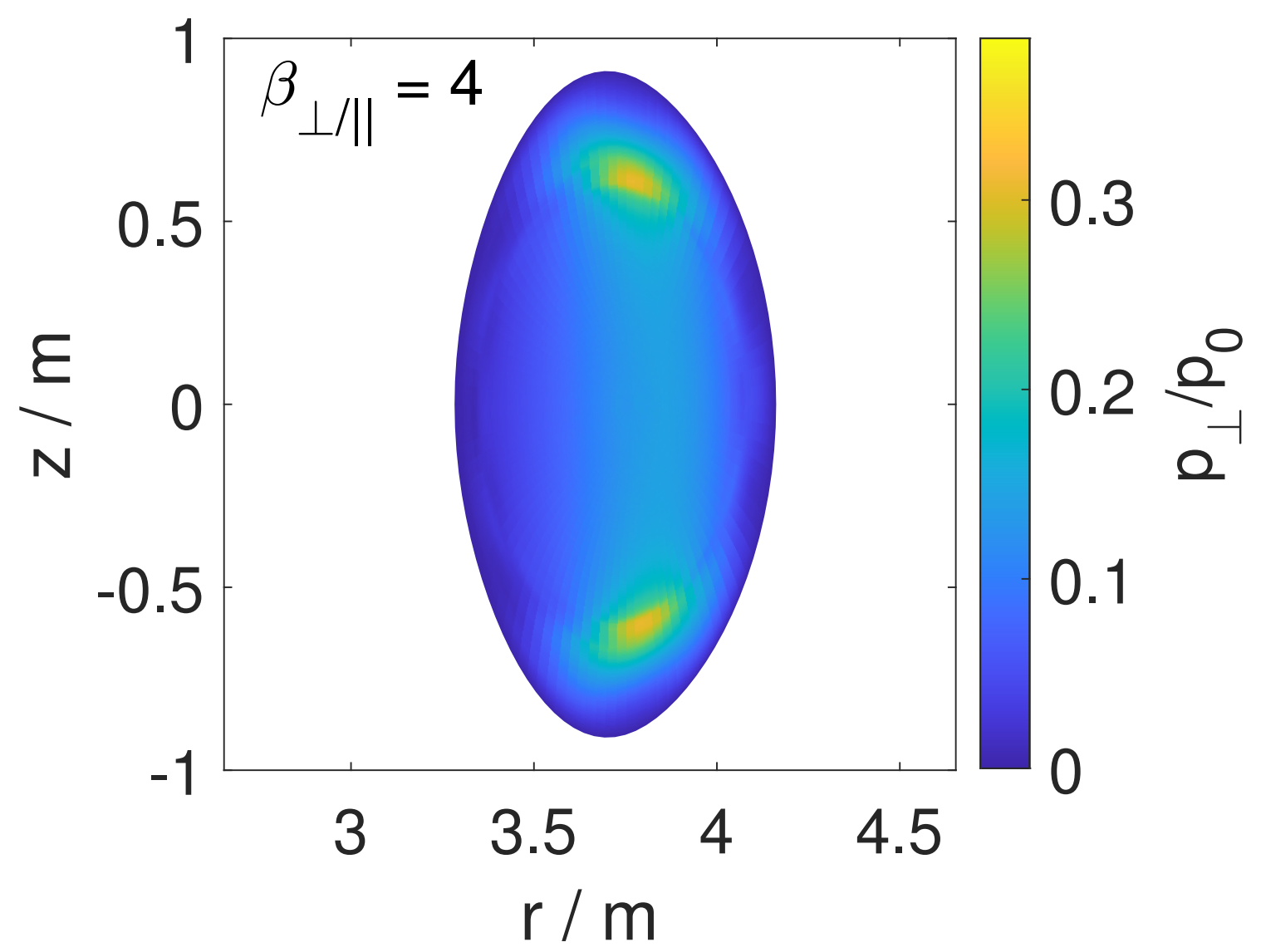

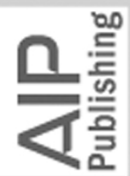




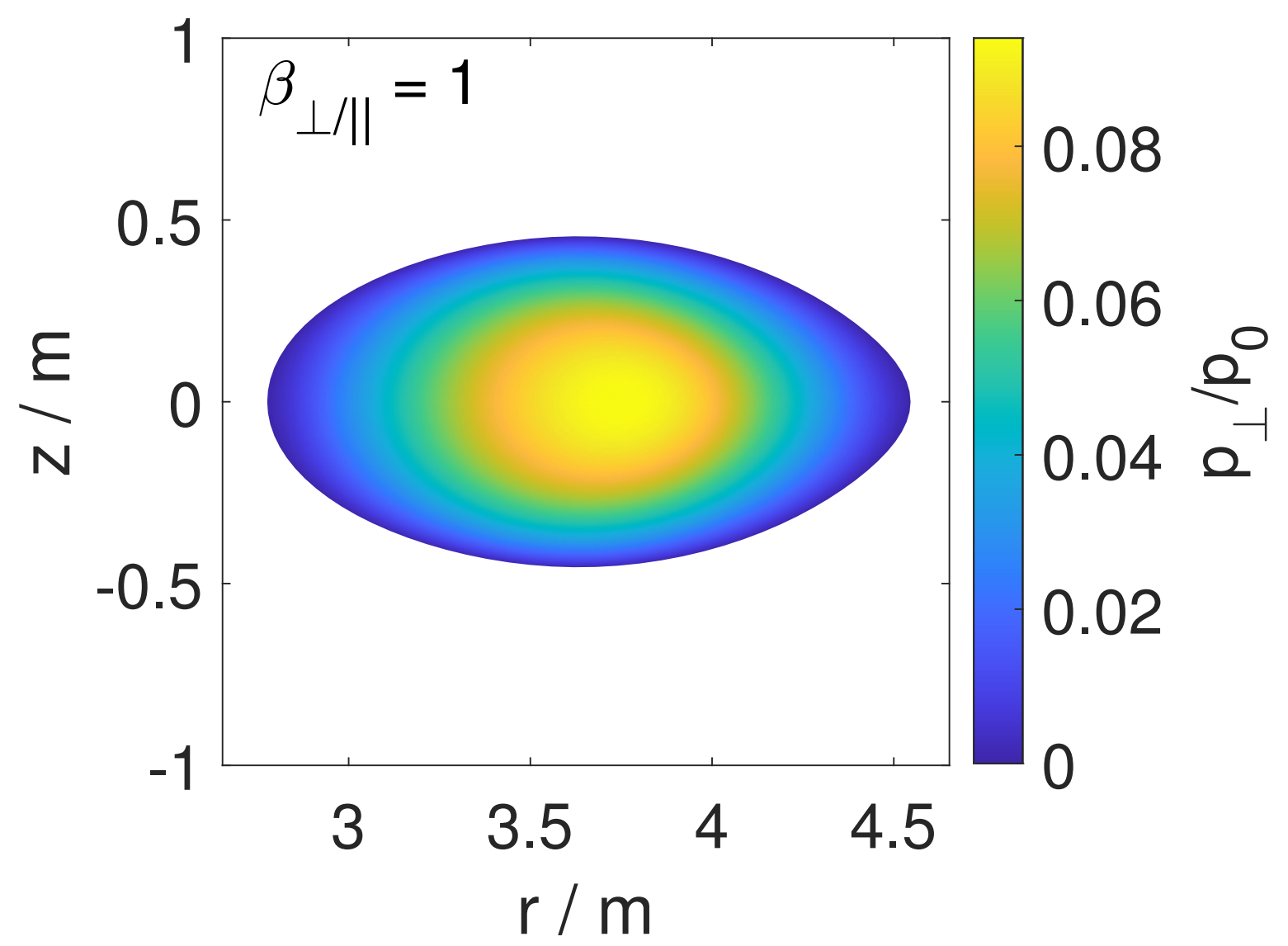

은 


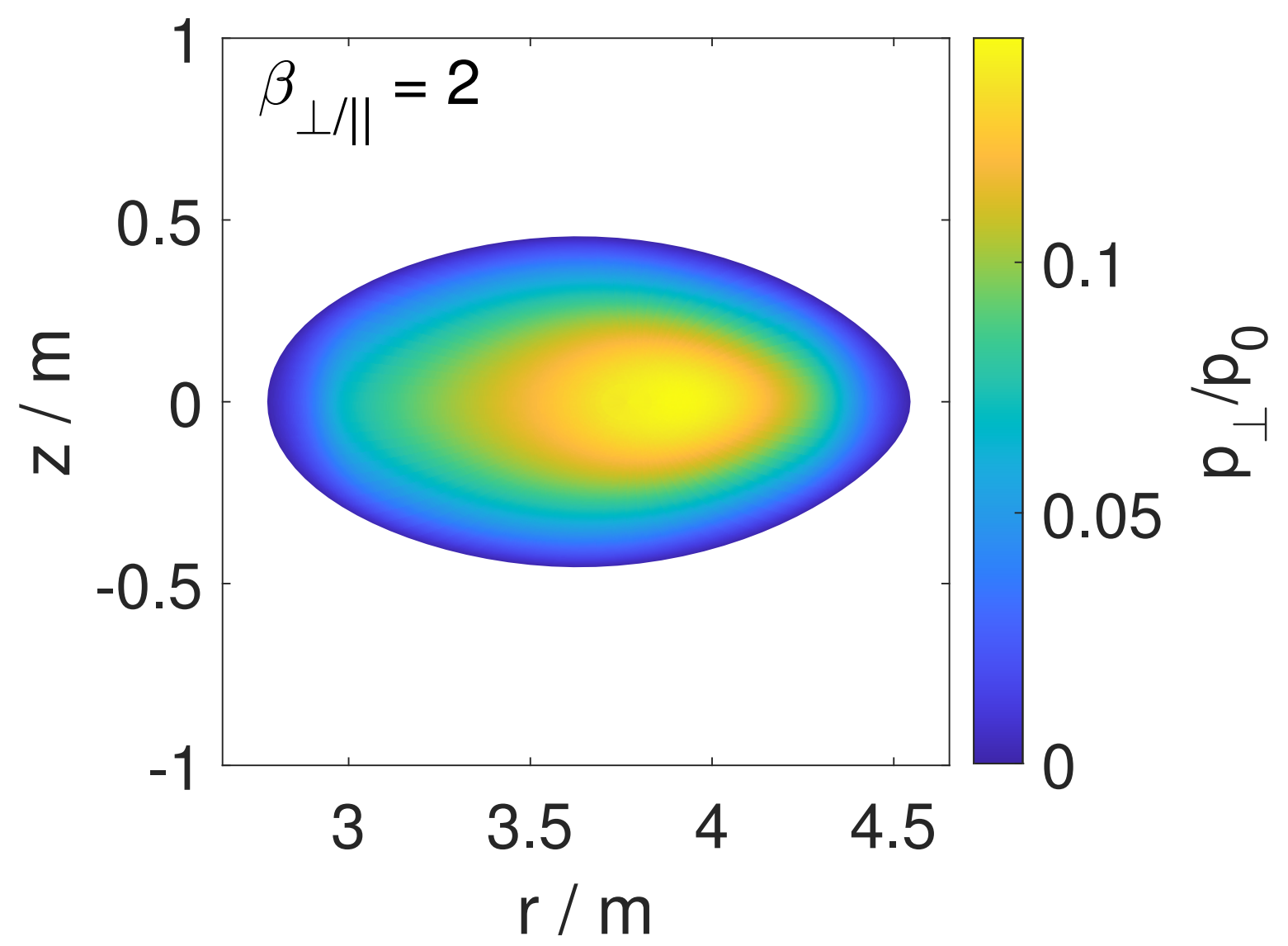

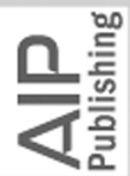




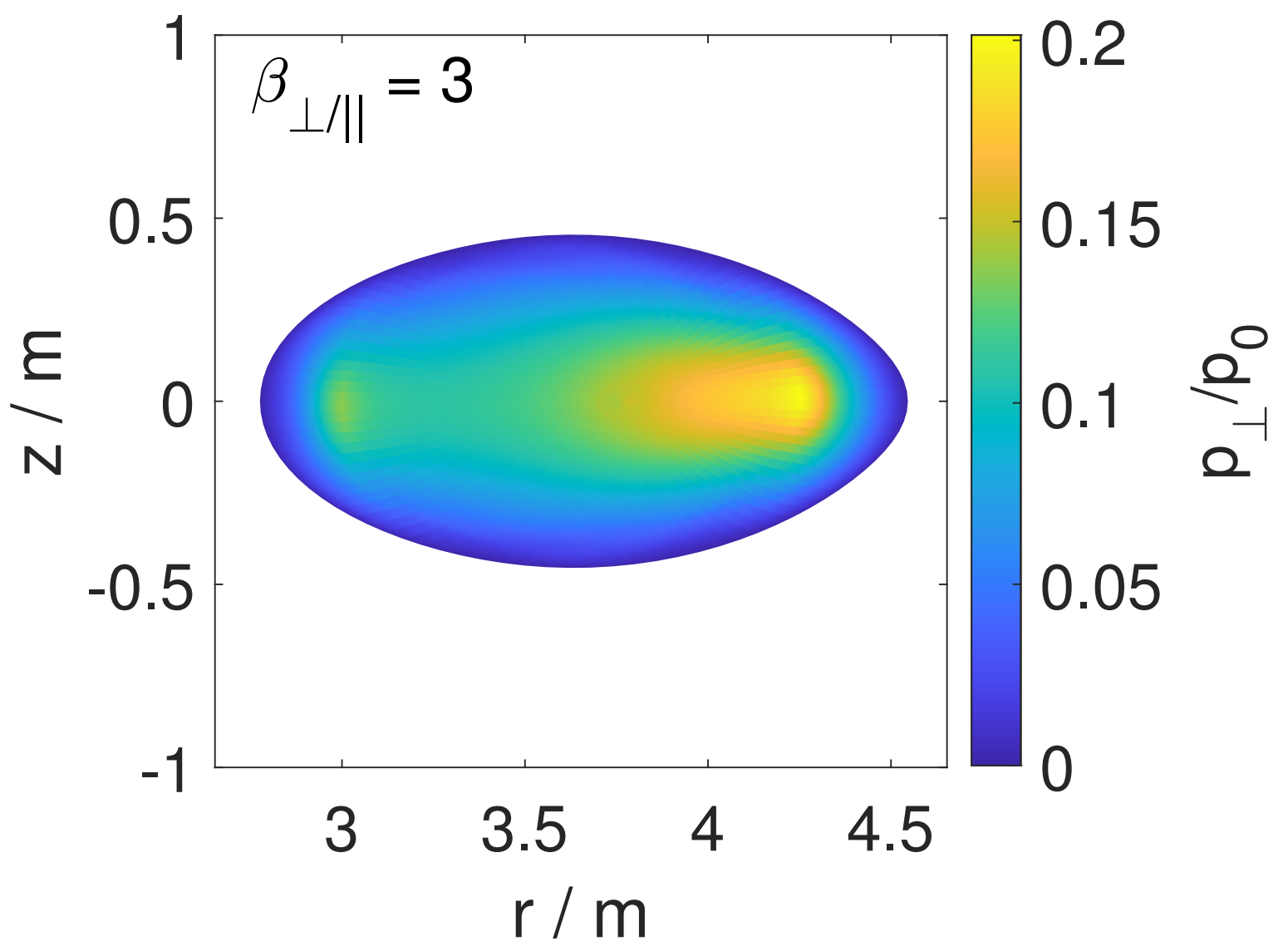

는 


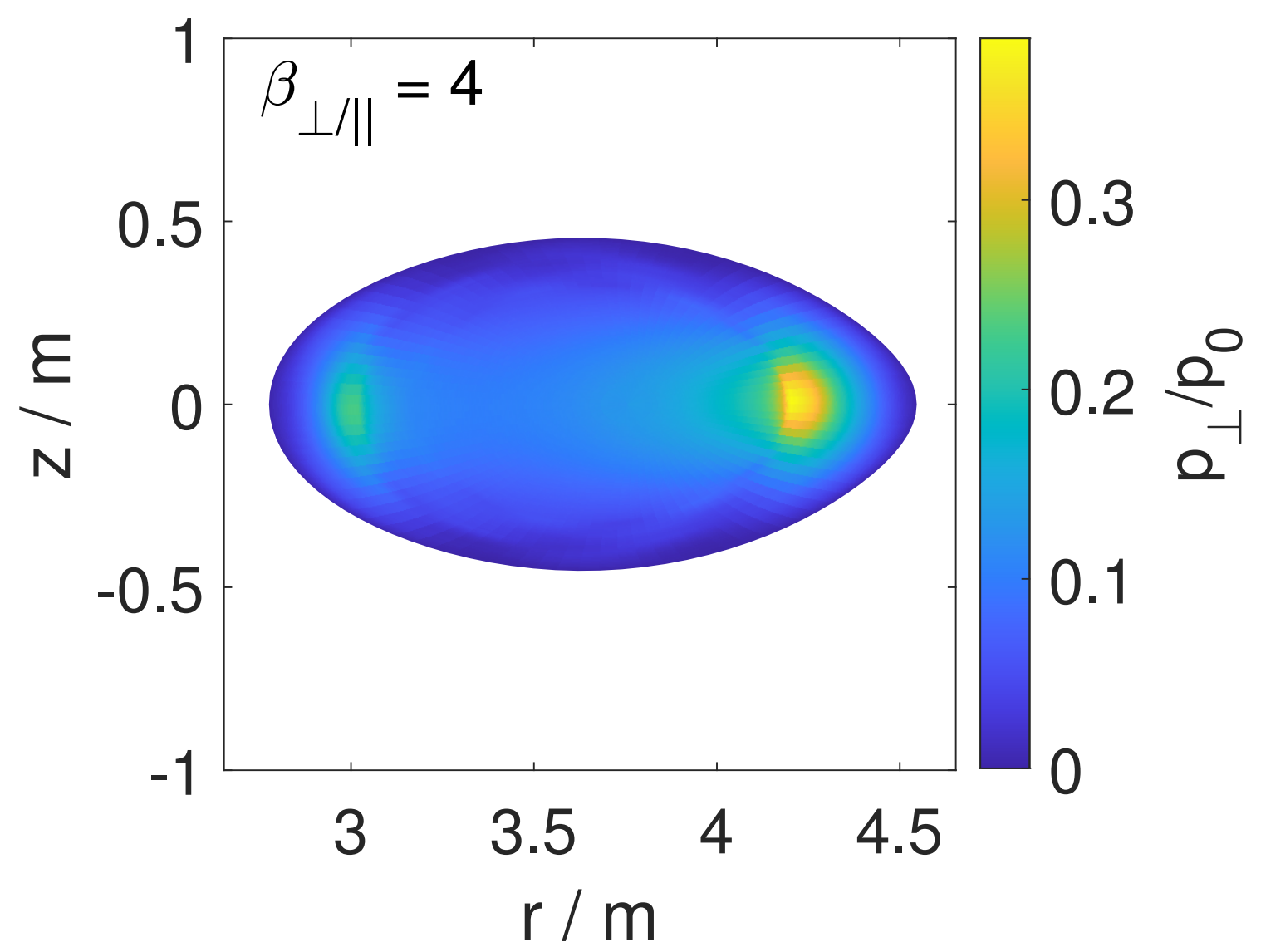

은 


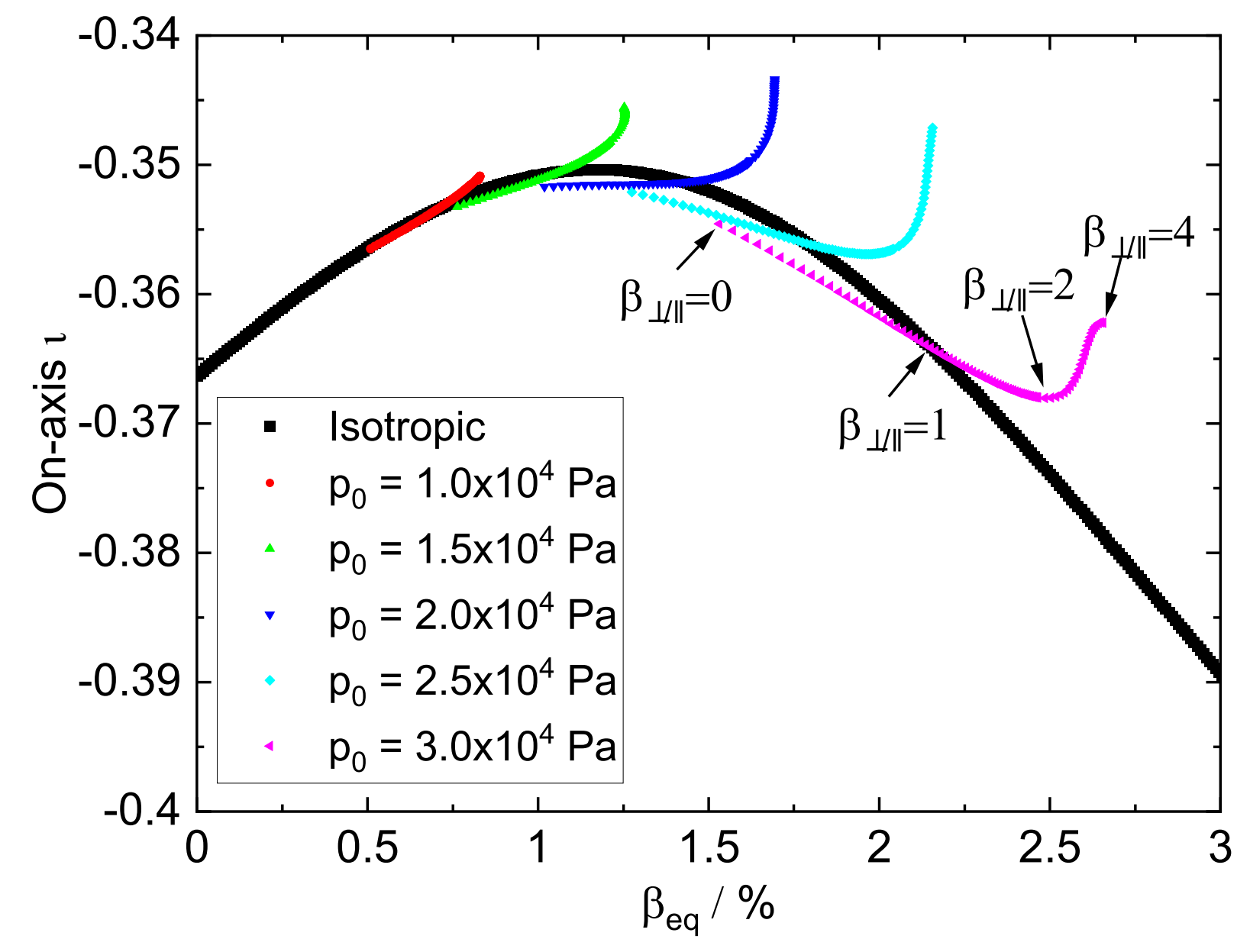




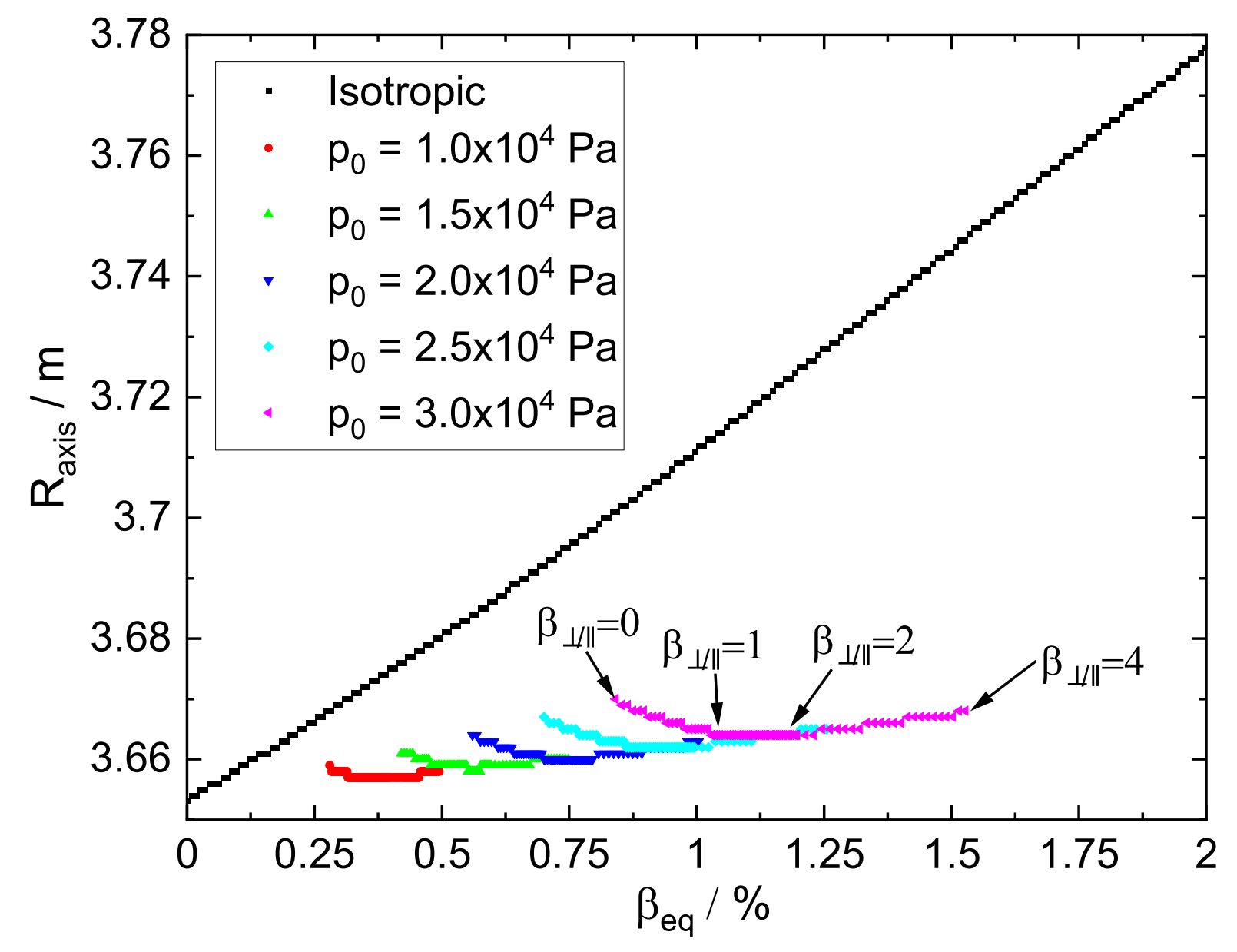




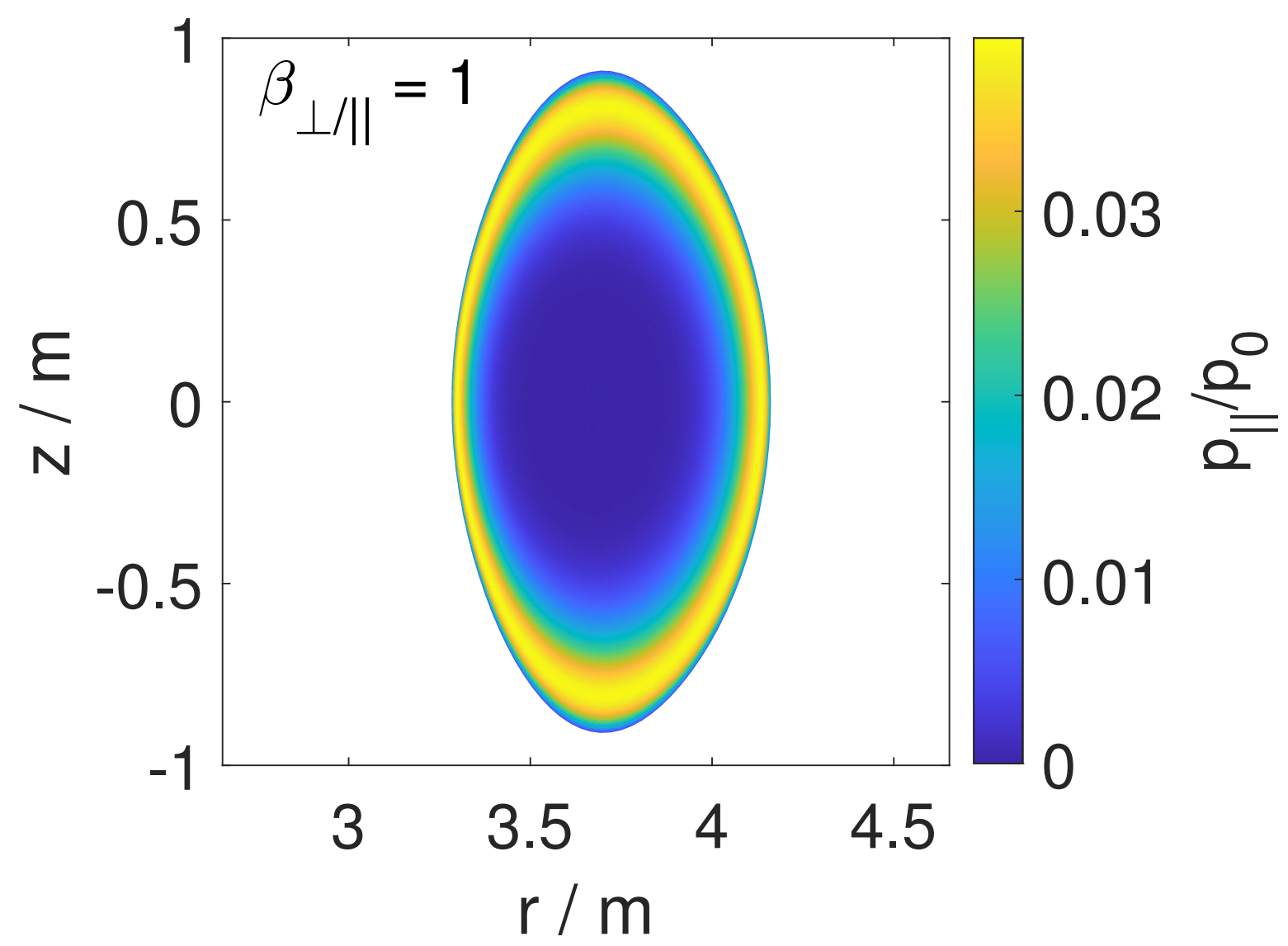

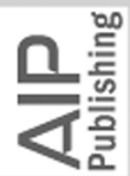




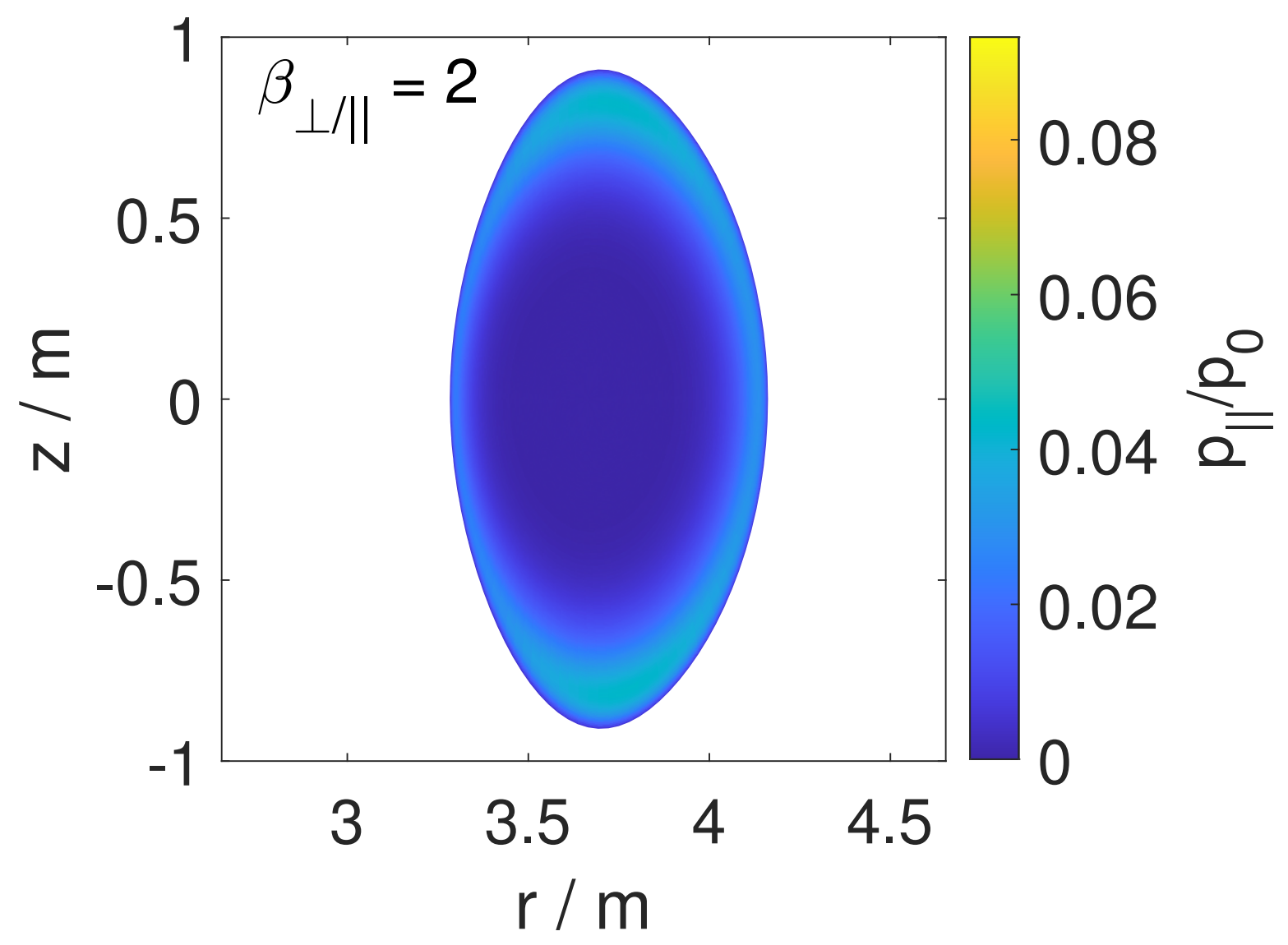

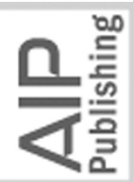




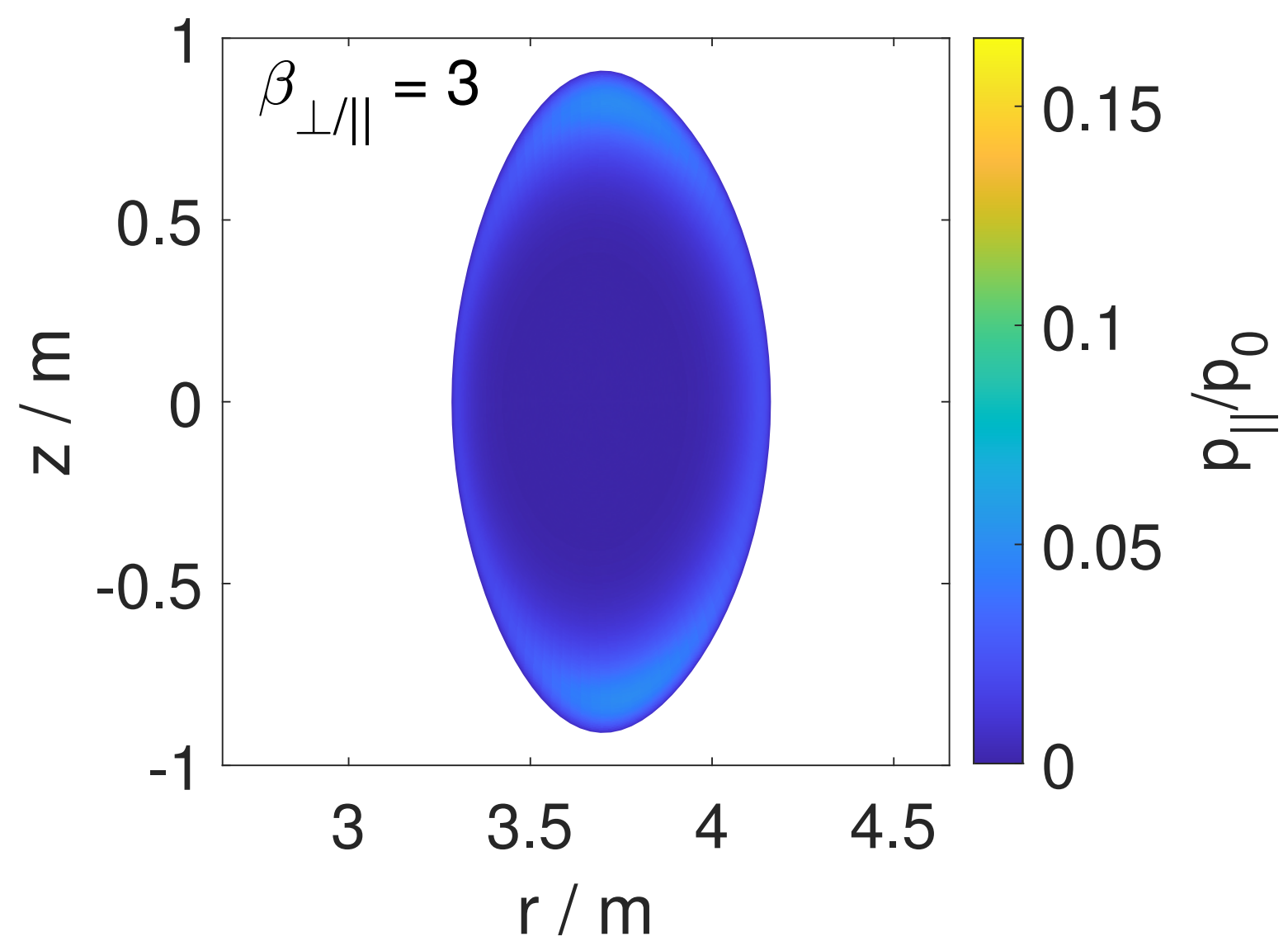

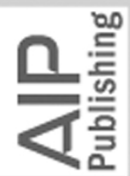




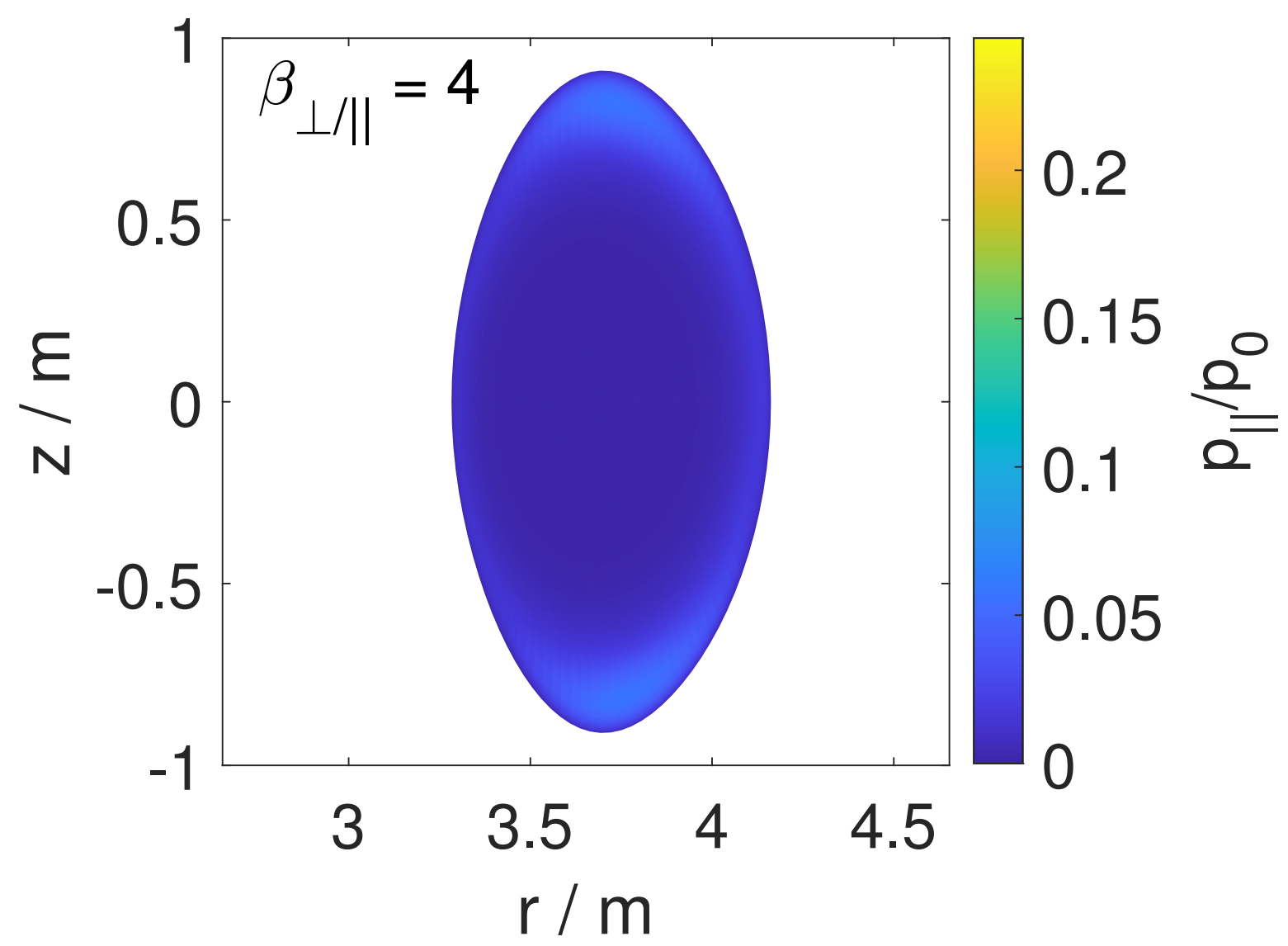

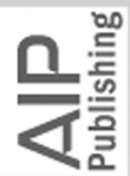




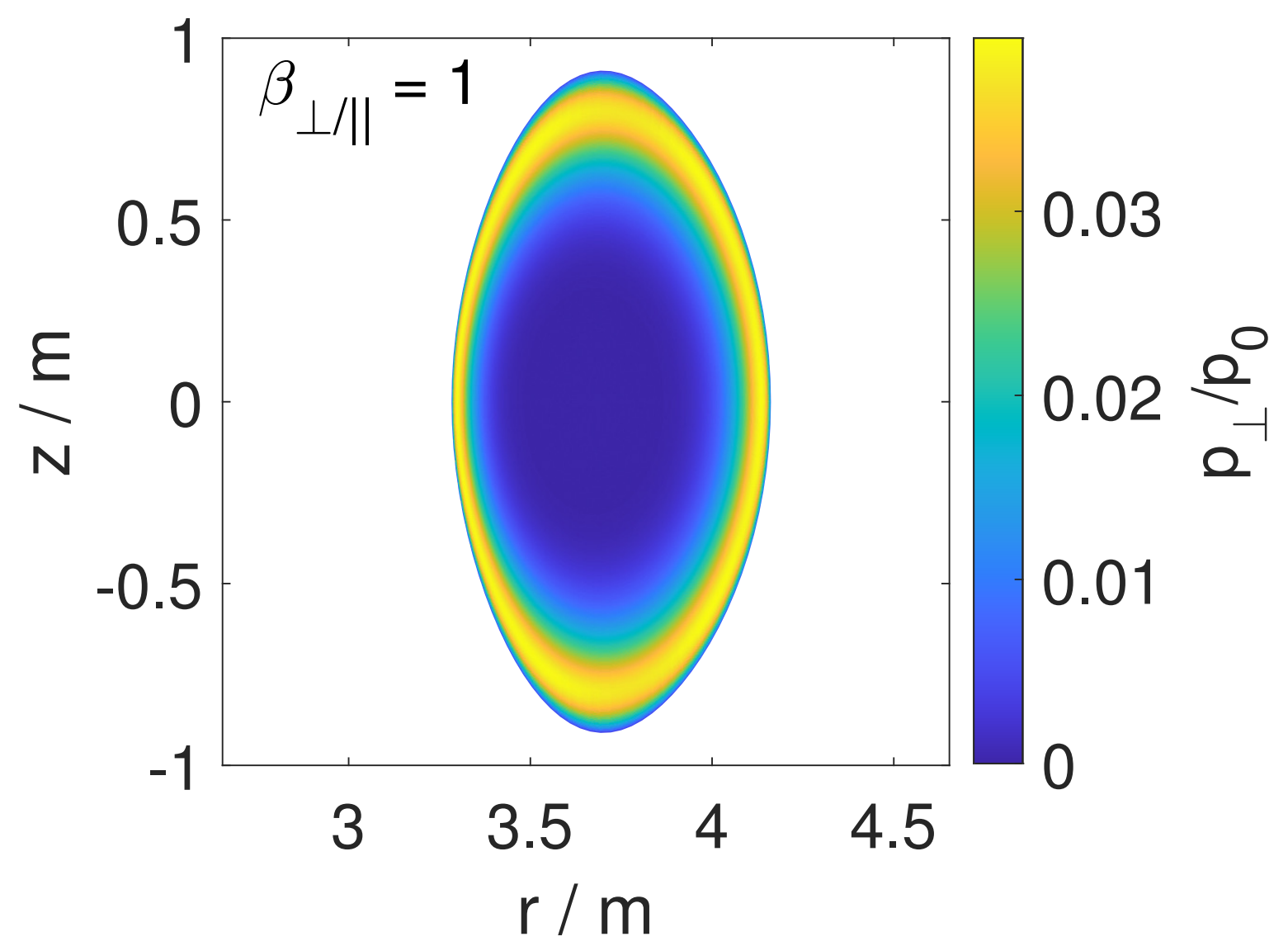

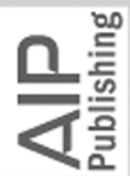




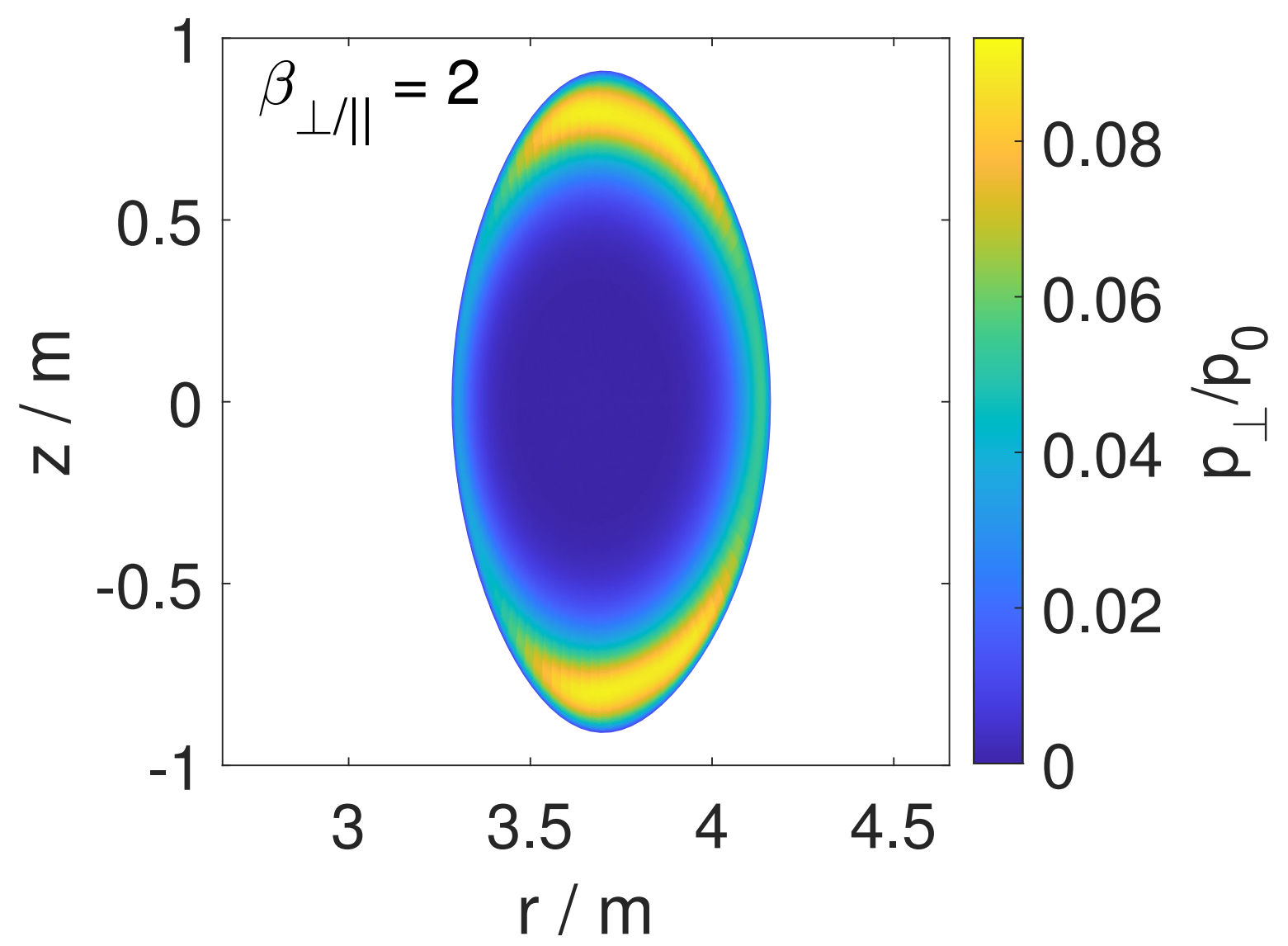

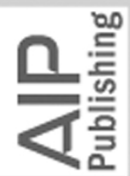




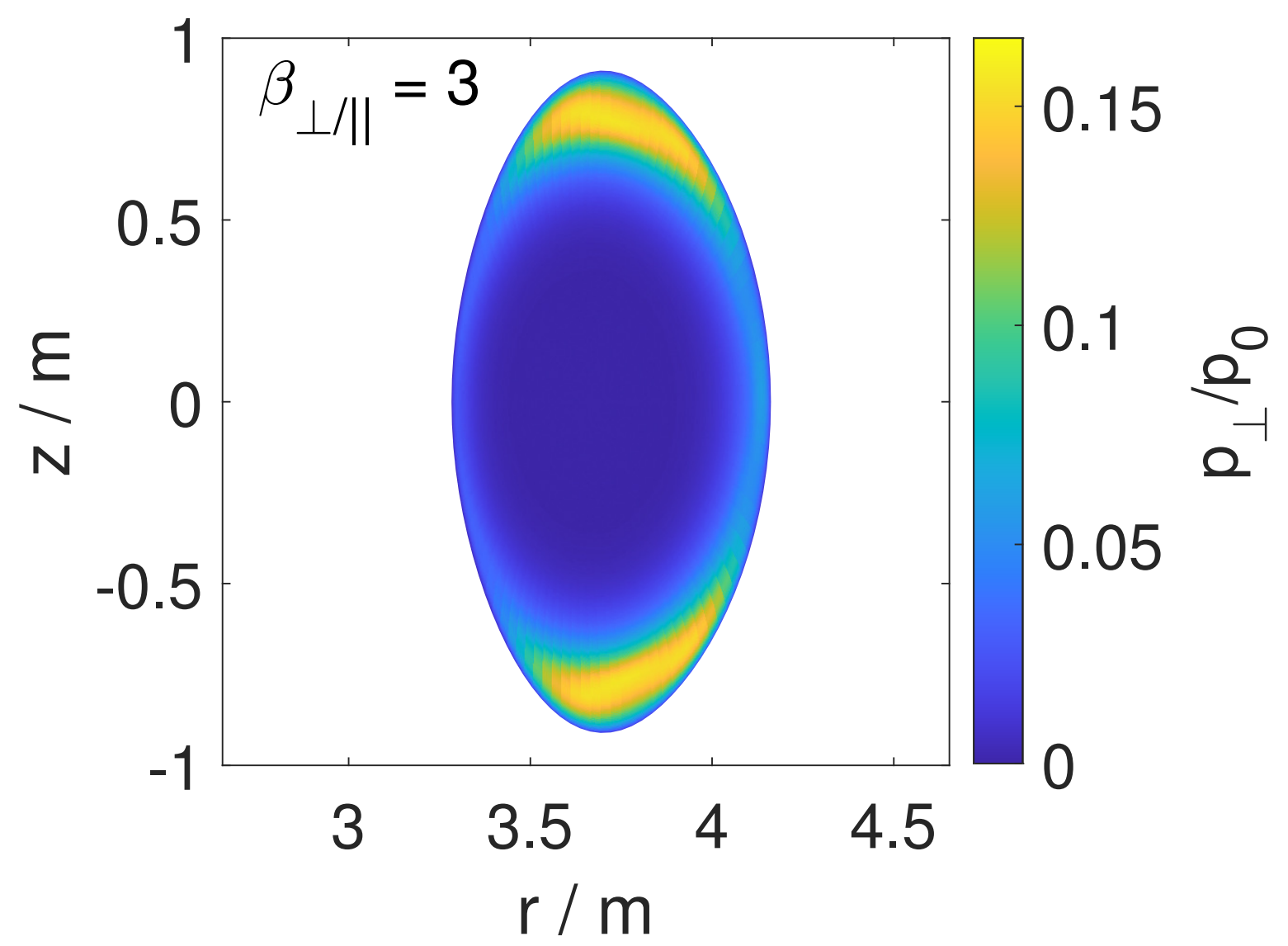

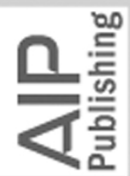




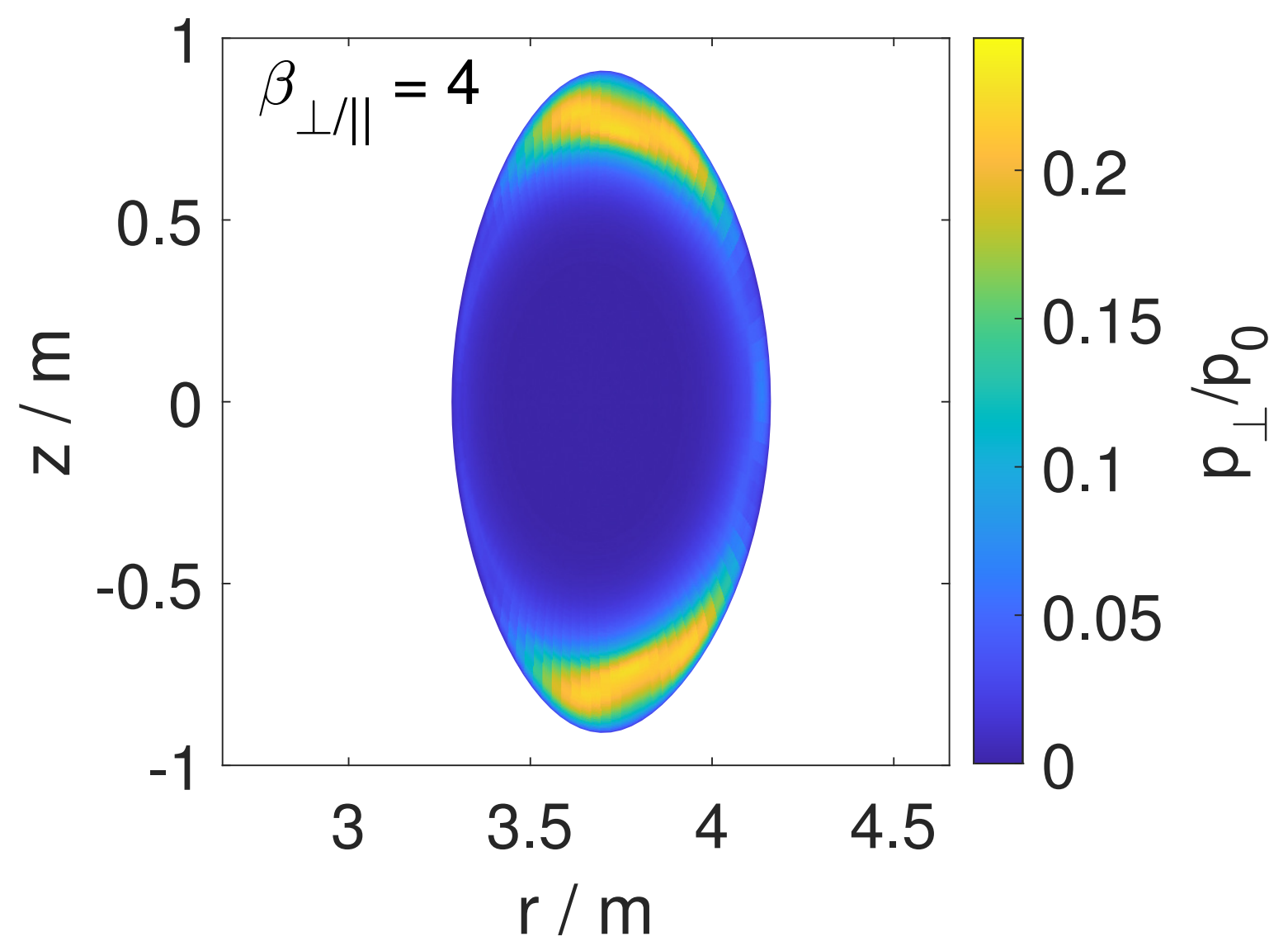

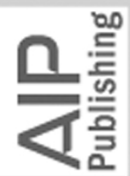




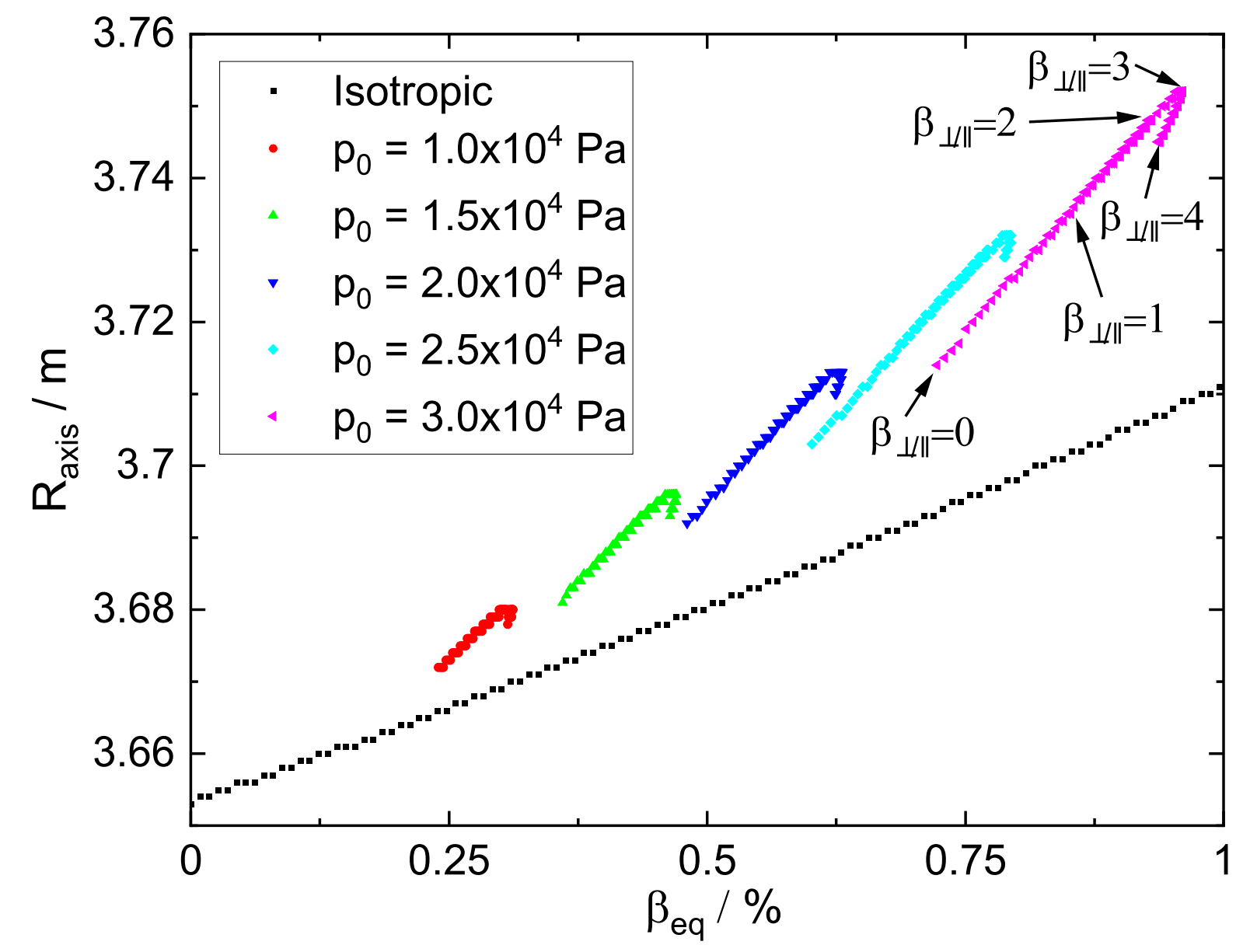




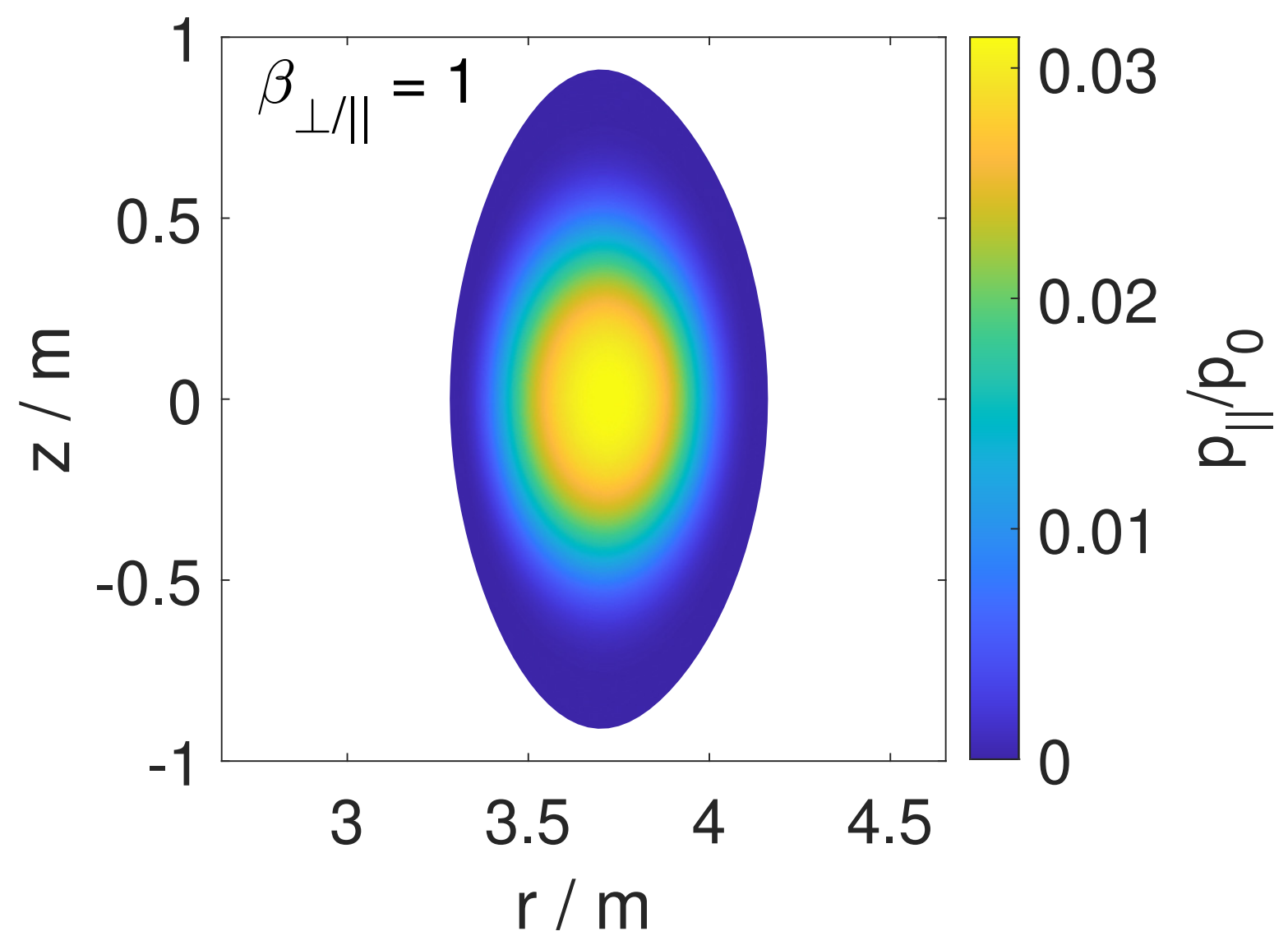

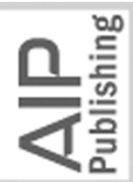




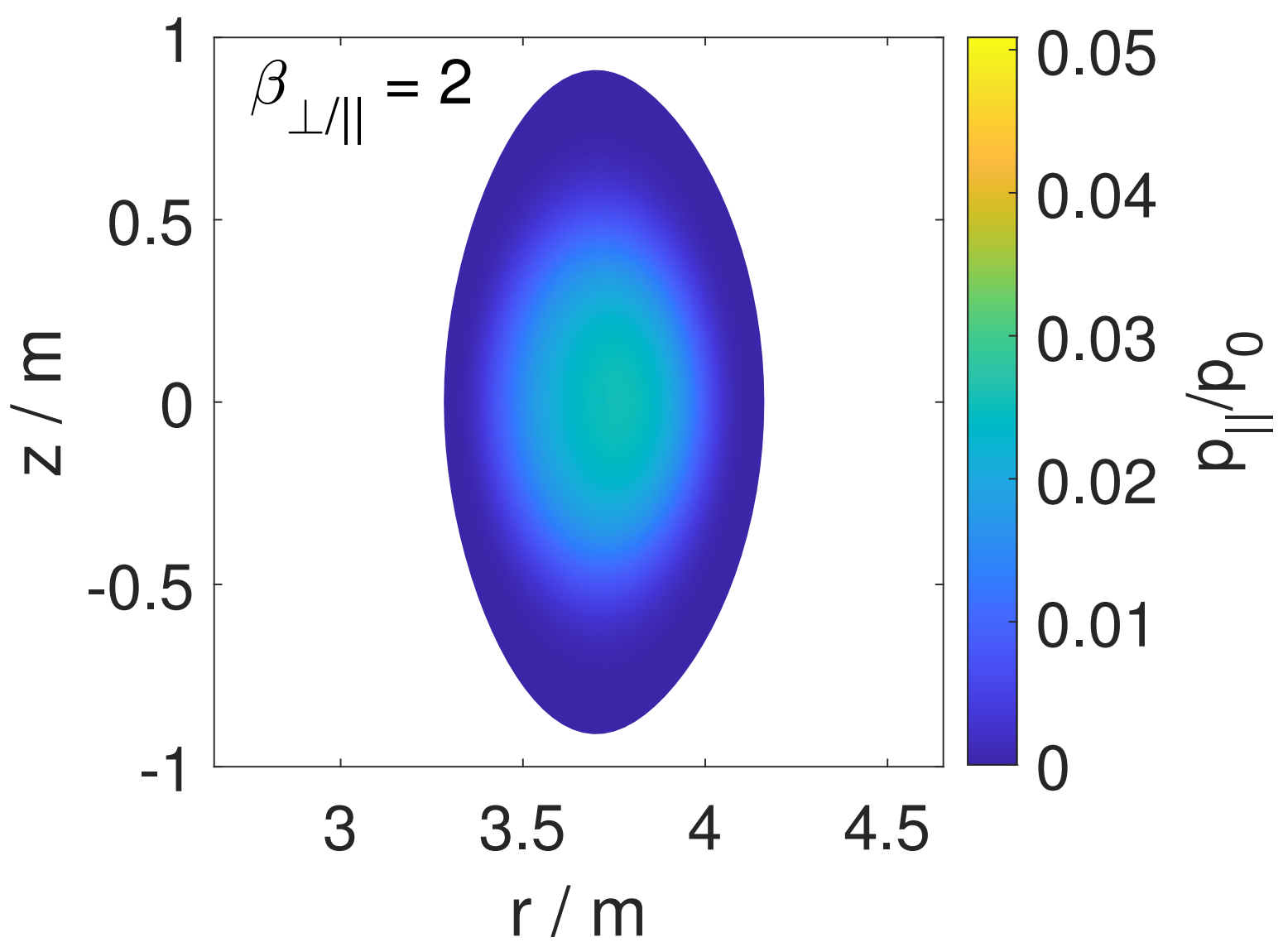

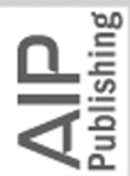




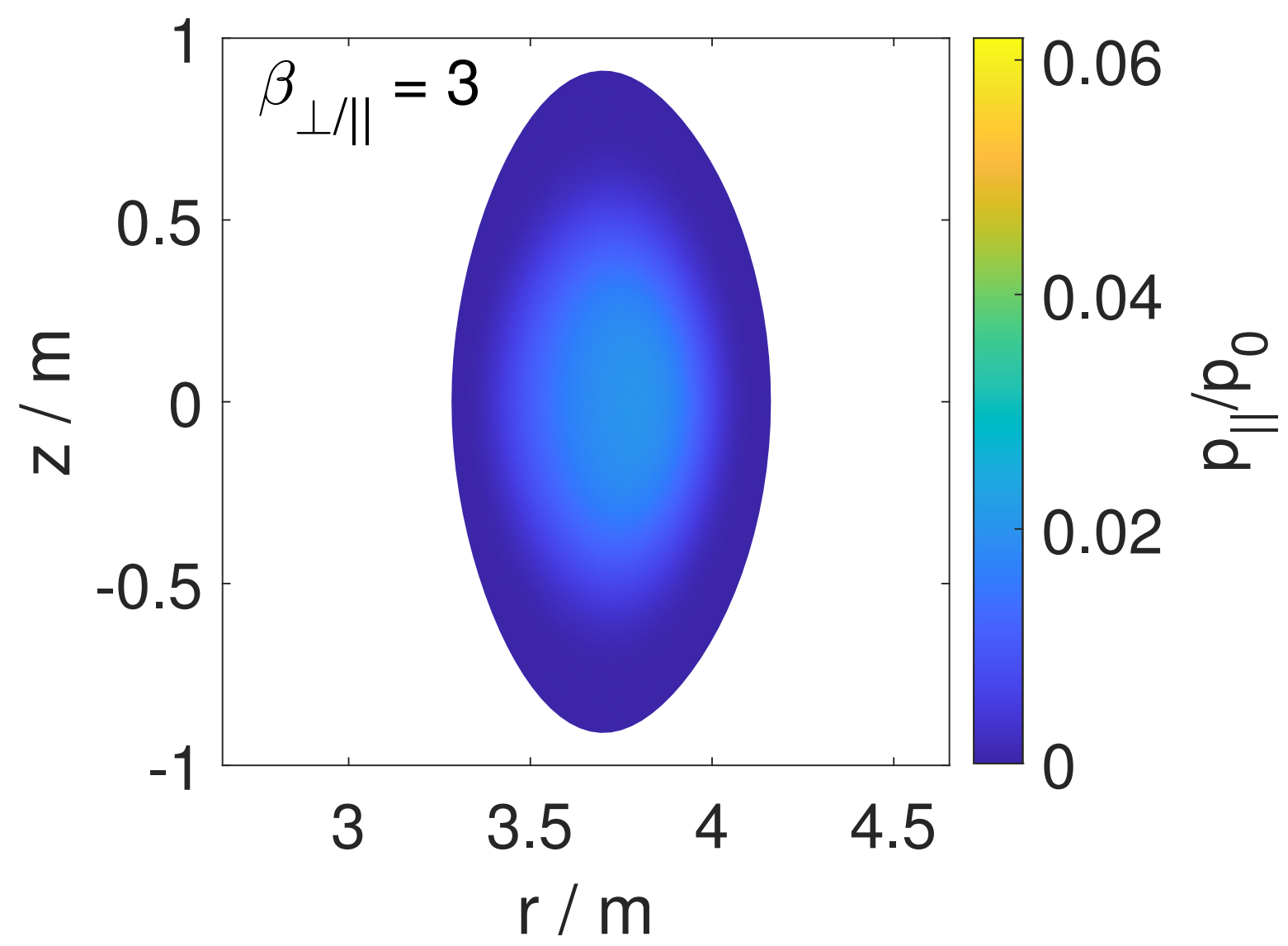

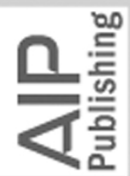




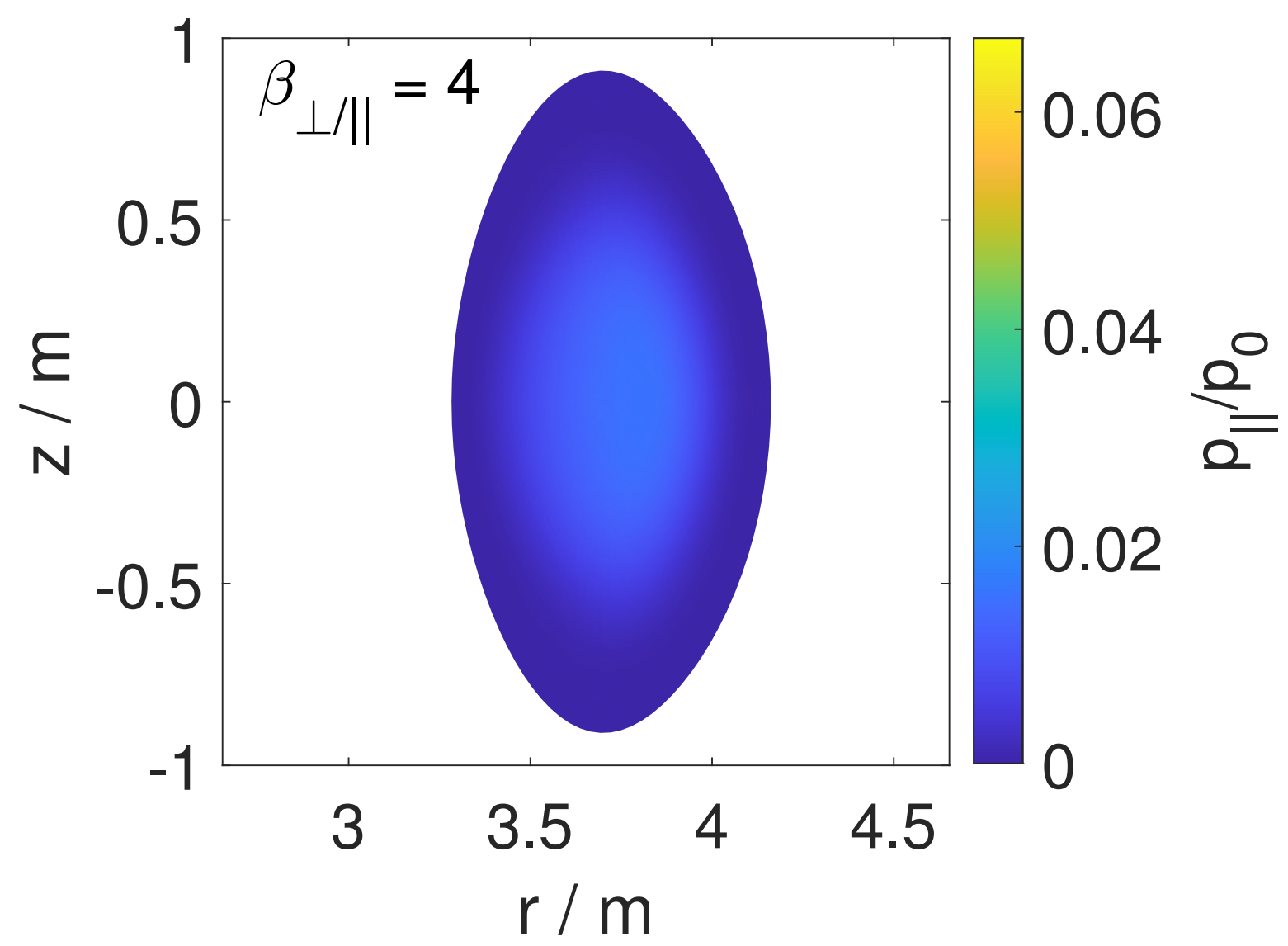

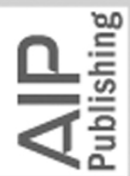




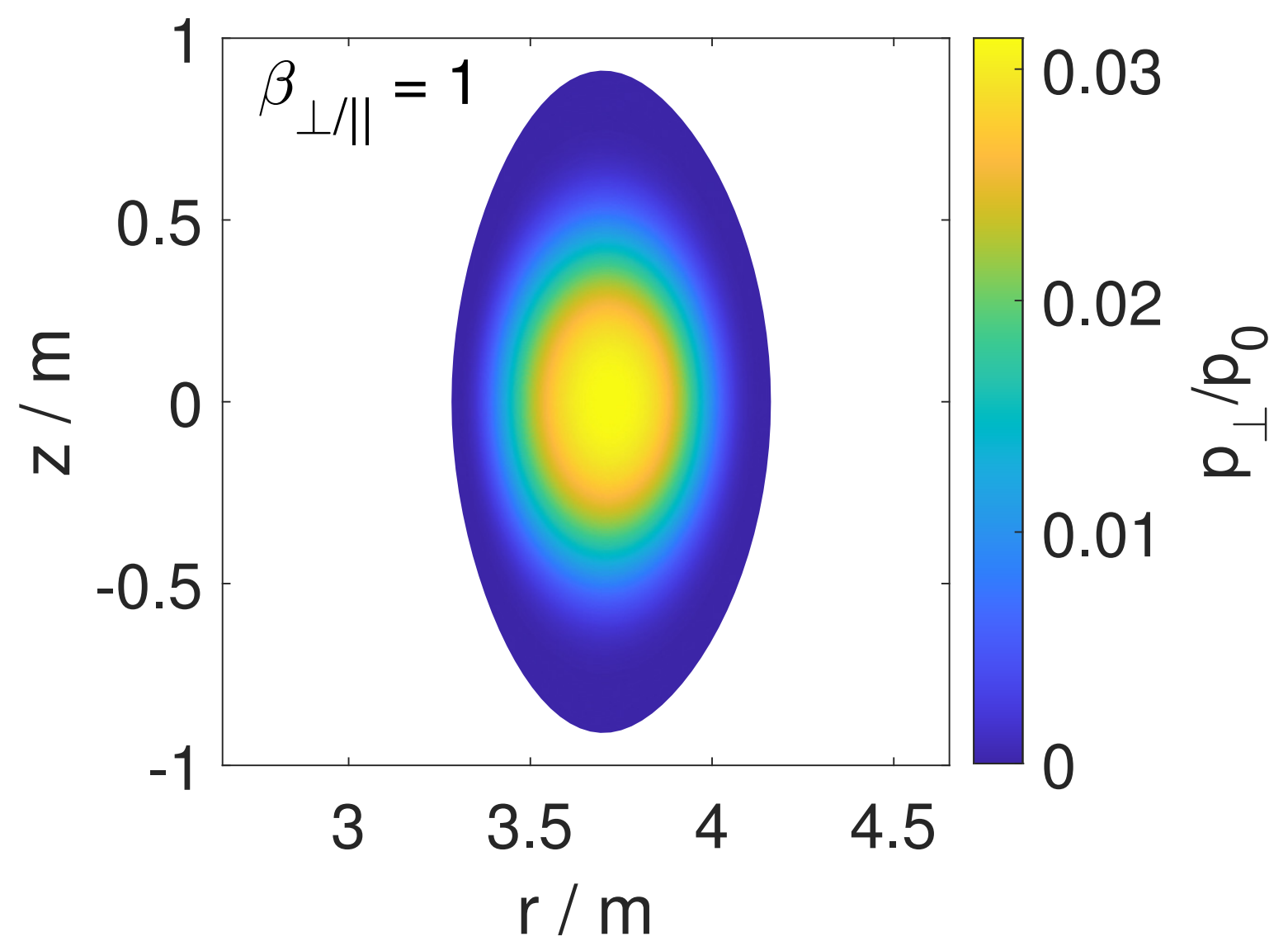

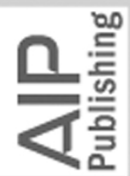




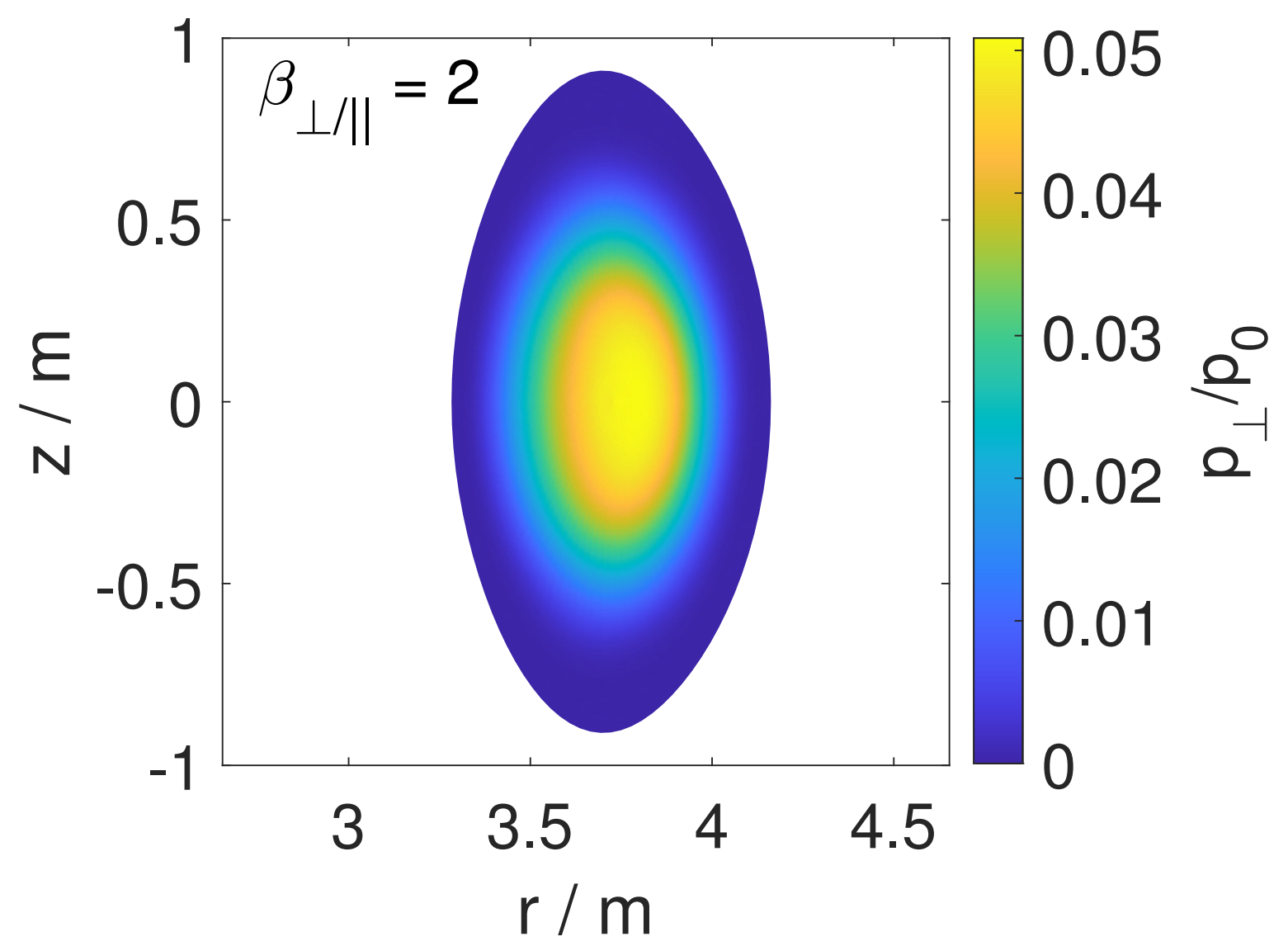

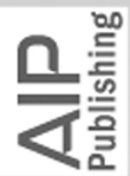




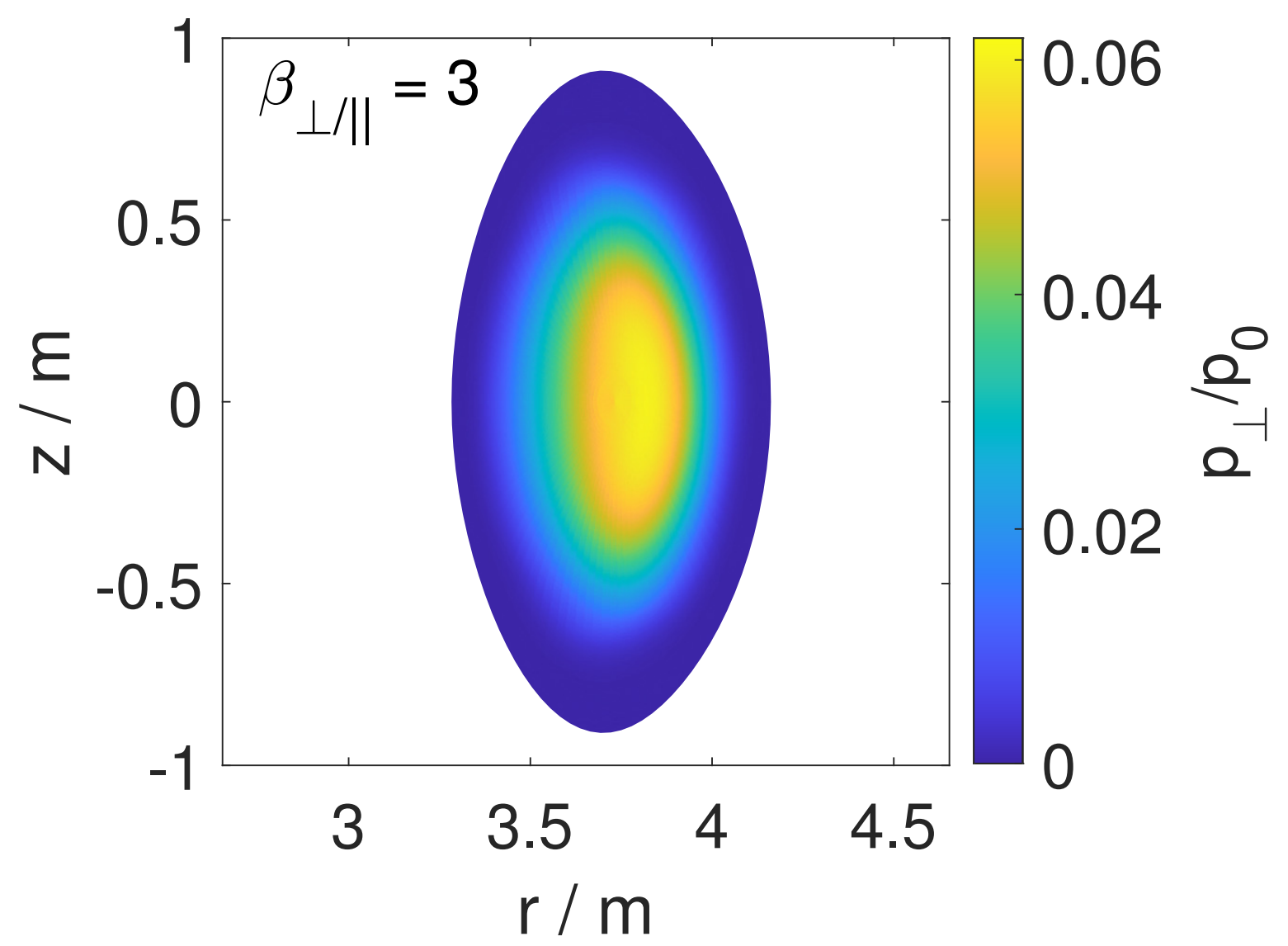

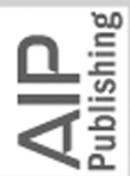




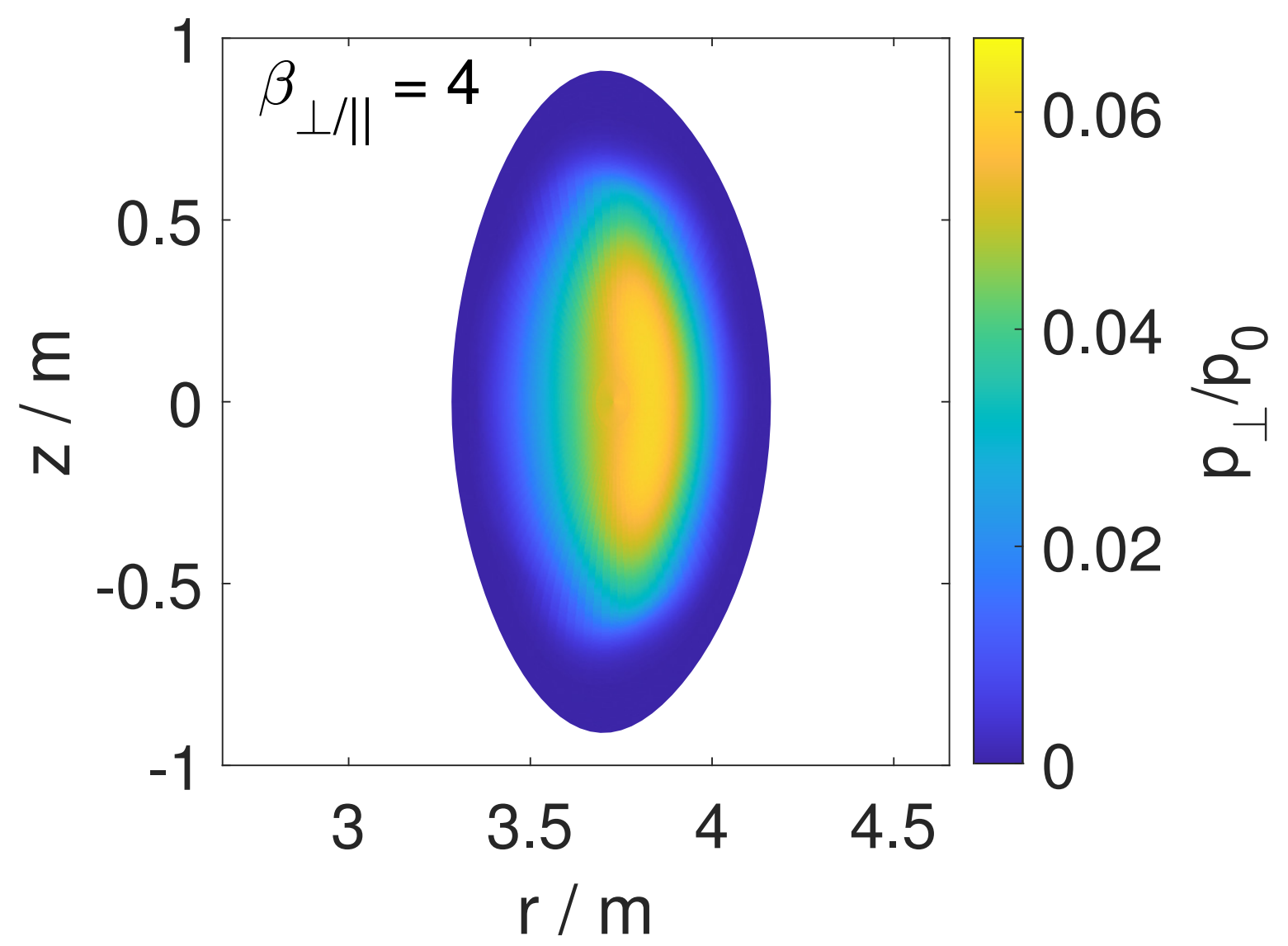

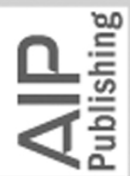




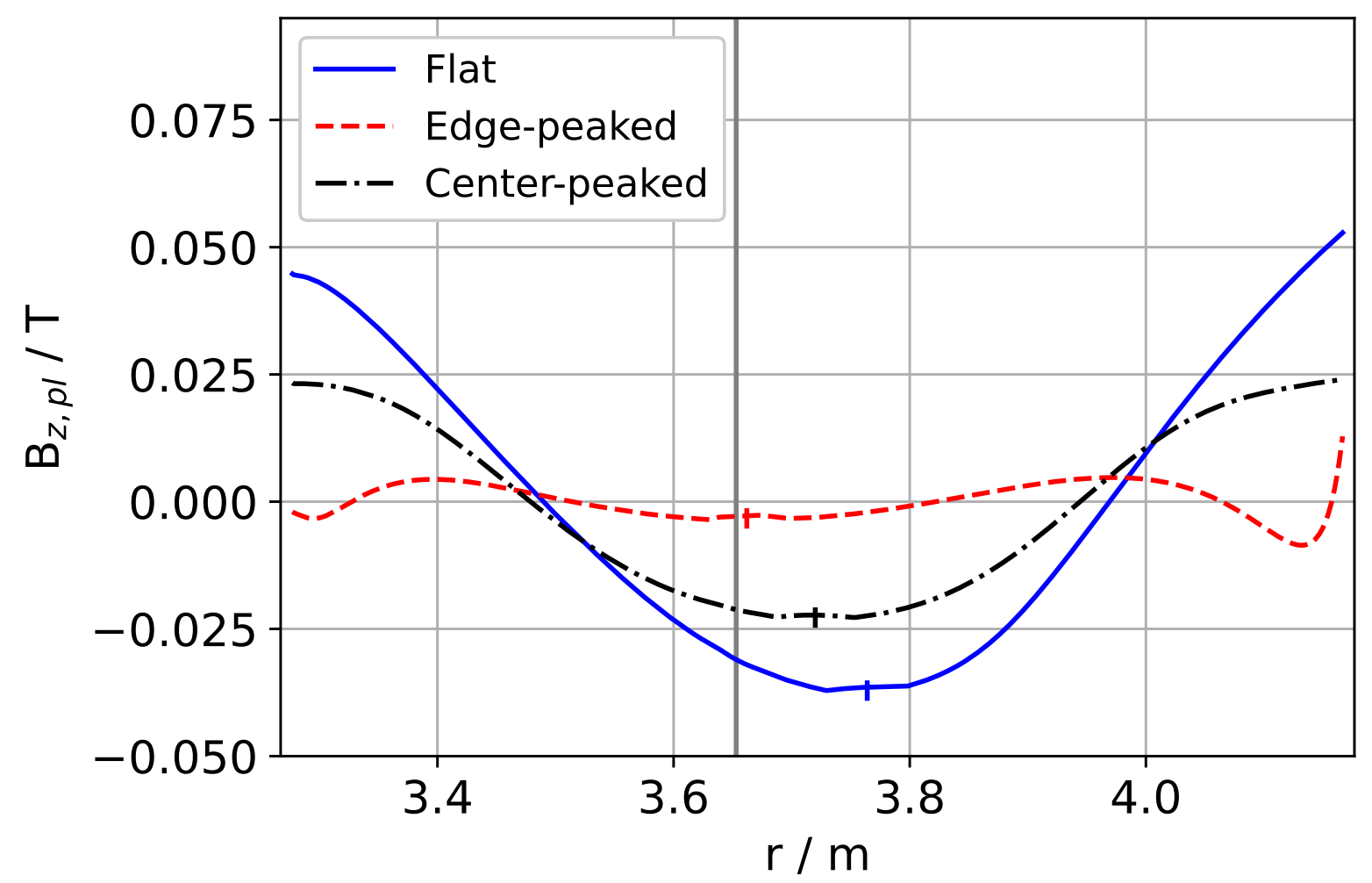

兄旁言 


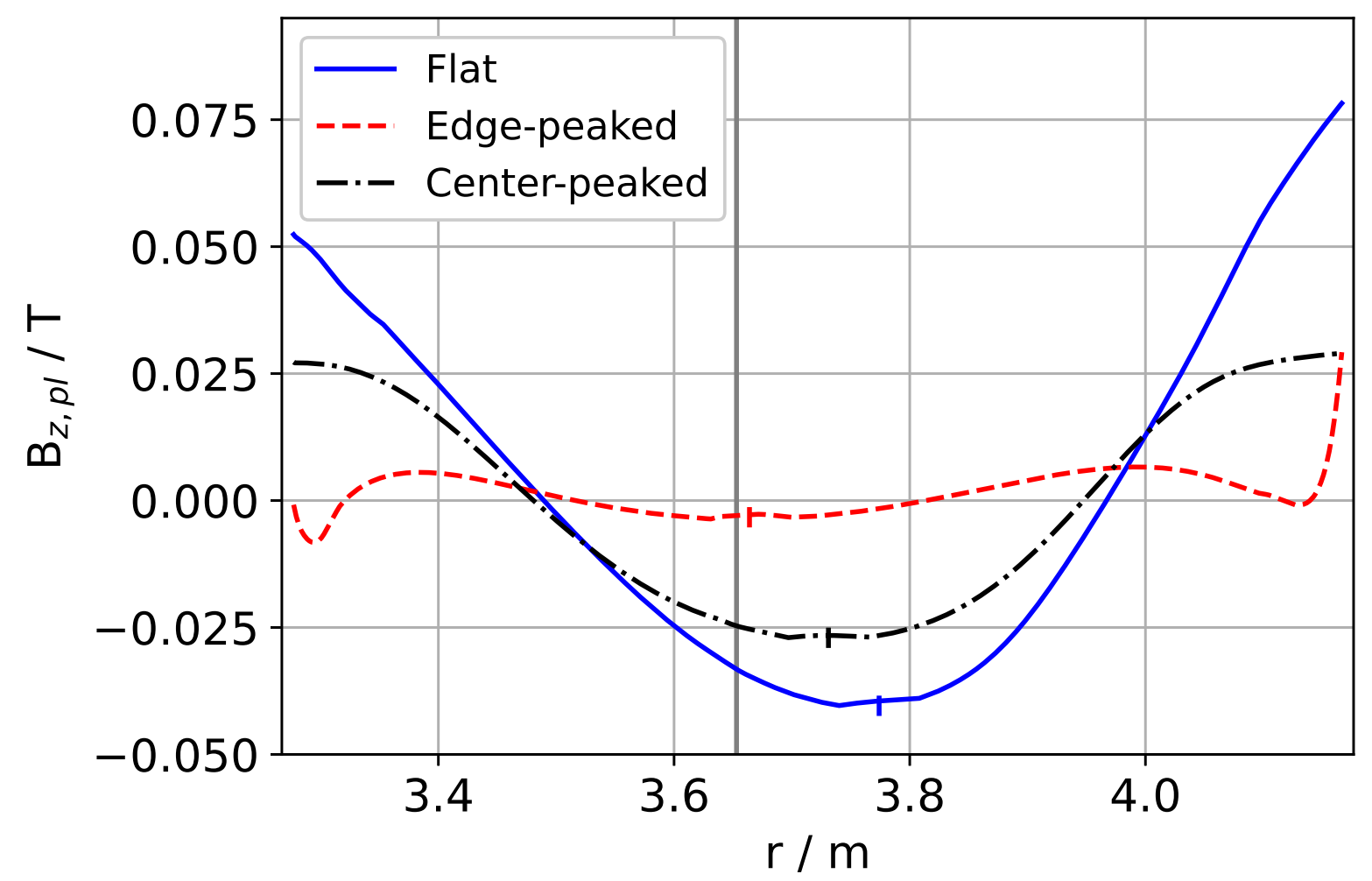

는 